\title{
THICK POINTS FOR SPATIAL BROWNIAN MOTION: MULTIFRACTAL ANALYSIS OF OCCUPATION MEASURE
}

\author{
By Amir Dembo, ${ }^{1}$ Yuval Peres, ${ }^{2}$ Jay Rosen $^{3}$ And Ofer Zeitouni ${ }^{4}$ \\ Stanford University, University of California, Berkeley, \\ College of Staten Island, CUNY and Technion
}

Let $\mathscr{T}(x, r)$ denote the total occupation measure of the ball of radius $r$ centered at $x$ for Brownian motion in $\mathbb{R}^{3}$. We prove that $\sup _{|x| \leq 1} \mathscr{T}(x, r) /\left(r^{2}\right.$ $|\log r|) \rightarrow 16 / \pi^{2}$ a.s. as $r \rightarrow 0$, thus solving a problem posed by Taylor in 1974. Furthermore, for any $a \in\left(0,16 / \pi^{2}\right)$, the Hausdorff dimension of the set of "thick points" $x$ for which $\lim \sup _{r \rightarrow 0} \mathscr{T}(x, r) /\left(r^{2}|\log r|\right)=a$ is almost surely $2-a \pi^{2} / 8$; this is the correct scaling to obtain a nondegenerate "multifractal spectrum" for Brownian occupation measure. Analogous results hold for Brownian motion in any dimension $d>3$. These results are related to the LIL of Ciesielski and Taylor for the Brownian occupation measure of small balls in the same way that Lévy's uniform modulus of continuity, and the formula of Orey and Taylor for the dimension of "fast points" are related to the usual LIL. We also show that the lim inf scaling of $\mathscr{T}(x, r)$ is quite different: we exhibit nonrandom $c_{1}, c_{2}>0$, such that $c_{1}<\sup _{x} \liminf \operatorname{in}_{r \rightarrow 0} \mathscr{T}(x, r) / r^{2}<c_{2}$ a.s. In the course of our work we provide a general framework for obtaining lower bounds on the Hausdorff dimension of random fractals of "limsup type."

1. Introduction. For any Borel measurable function $f$ from $0 \leq t \leq T$ to $\mathbb{R}^{d}$ we denote by $\mu_{T}^{f}$ its occupation measure,

$$
\mu_{T}^{f}(A)=\int_{0}^{T} \mathbf{1}_{A}\left(f_{t}\right) d t
$$

for all Borel sets $A \subseteq \mathbb{R}^{d}$. Throughout, $B(x, r)$ denotes the ball in $\mathbb{R}^{d}$ of radius $r$ centered at $x$, and $\left\{W_{t}\right\}_{t \geq 0}$ denotes Brownian motion in $\mathbb{R}^{d}, d \geq 3$.

In the last decade, much insight into the structure of various measures has been gained from their multifractal analysis. A general introduction to this analysis can be found in [8], [12] and [22]; certain important random measures are analyzed in [9], [11], [20], [24] and [28].

Consider Brownian occupation measure $\mu_{T}^{W}$ in $\mathbb{R}^{d}, d \geq 3$. It is well known that for almost all Brownian paths $W$, the pointwise Hölder exponent

$$
\operatorname{Hölder}\left(\mu_{T}^{W}, x\right):=\lim _{\varepsilon \rightarrow 0} \frac{\log \mu_{T}^{W}(B(x, \varepsilon))}{\log \varepsilon}
$$

Received August 1998; revised July 1999.

${ }^{1}$ Supported in part by NSF Grant DMS-97-04552 and by a US-Israel BSF grant.

${ }^{2}$ Supported in part by NSF Grants DMS-94-04391 and DMS-98-03597.

${ }^{3}$ Supported in part by grants from NSF and PSC-CUNY.

${ }^{4}$ Supported in part by MSRI and by US-Israel BSF grant.

AMS 1991 subject classifications. Primary 60J65, 28A80; secondary 60J55, 60F99, 28A78.

Key words and phrases. thick points, multifractal analysis, occupation measure. 
takes the value 2 for all points $x$ in the range $\left\{W_{t} \mid 0 \leq t \leq T\right\}$. In particular, the usual multifractal spectrum $a \mapsto \operatorname{dim}\left\{x \in \mathbb{R}^{d}: \operatorname{Hölder}\left(\mu_{T}^{W}, x\right)=a\right\}$ vanishes for all $a \neq 2, a>0$. Indeed, this fact was crucial in Kaufman's work [10], written long before the term "multifractal" was invented.

Rather than being the end of the story, this means that standard multifractal analysis must be refined to capture the delicate fluctuations of occupation measure under scaling; the problem of obtaining such a refined analysis was posed by $\mathrm{Hu}$ and Taylor in 1997 ([9], page 287), but it is closely linked to problems posed by Taylor [26] in 1974. Our main results, Theorems 1.1 and 1.3 , resolve these problems.

The correct scaling for studying the fluctuations of occupation measure was already indicated in [26]; more details were given by Perkins and Taylor ([18], Lemmas 2.3 and 2.5), who showed that there exist absolute constants $0<$ $c_{1}<c_{2}<\infty$, such that almost surely for all points $x \in\left\{W_{t} \mid 0 \leq t \leq T\right\}$ and all positive $\varepsilon \leq \varepsilon_{0}(\omega)$,

$$
c_{1} \varepsilon^{2} /|\log \varepsilon| \leq \mu_{T}^{W}(B(x, \varepsilon)) \leq c_{2} \varepsilon^{2}|\log \varepsilon| .
$$

(As they point out, the lower bound is immediate from Lévy's uniform modulus of continuity.)

Our main result describes the multifractal nature, in a fine scale, of "thick points" for the occupation measure of Brownian motion in $\mathbb{R}^{d}, d \geq 3$. [We call a point $x \in \mathbb{R}^{d}$ on the Brownian path a thick point if $x$ is in the set considered in (1.3) for some $a>0$; similiarly, $t>0$ is called a thick time if it is in the set Thick $_{a}$ considered in (1.4) for some $a>0$ and $T>0$.]

THEOREM 1.1. With $d \geq 3$, let $q_{d}$ denote the first positive zero of the Bessel function $J_{d / 2-2}(x)$. (See [31] for information on $q_{d}$; in particular, $q_{3}=\pi / 2$.) Then, for any $T \in(0, \infty]$ and all $0<a \leq 4 / q_{d}^{2}$,

$$
\operatorname{dim}\left\{x \in \mathbb{R}^{d} \mid \limsup _{\varepsilon \rightarrow 0} \frac{\mu_{T}^{W}(B(x, \varepsilon))}{\varepsilon^{2}|\log \varepsilon|}=a\right\}=2-a q_{d}^{2} / 2 \quad \text { a.s. }
$$

Equivalently, for any $T \in(0, \infty]$ and all $0<a \leq 4 / q_{d}^{2}$,

$$
\operatorname{dim}\left\{0 \leq t<T \mid \limsup _{\varepsilon \rightarrow 0} \frac{\mu_{T}^{W}\left(B\left(W_{t}, \varepsilon\right)\right)}{\varepsilon^{2}|\log \varepsilon|}=a\right\}=1-a q_{d}^{2} / 4 \quad \text { a.s. }
$$

Denote the set in (1.4) by Thick $_{a}$. Then Thick $\neq \neq \varnothing$ at the critical value $a=4 / q_{d}^{2}$.

For comparison purposes, recall three fundamental results on Brownian increments:

1. The large increments at a fixed time $t$ are governed by Khinchin's classical LIL,

$$
\limsup _{\varepsilon \rightarrow 0} \frac{W_{t+\varepsilon}-W_{t}}{(2 \varepsilon \log |\log \varepsilon|)^{1 / 2}}=1 \quad \text { a.s. }
$$


2. The dimension of certain exceptional fast points was determined by Orey and Taylor in [13]:

$$
\forall a \in[0,1] \quad \operatorname{dim}\left\{0 \leq t<T \mid \limsup _{\varepsilon \rightarrow 0} \frac{W_{t+\varepsilon}-W_{t}}{(2 \varepsilon|\log \varepsilon|)^{1 / 2}}=a\right\}=1-a^{2} \quad \text { a.s. }
$$

(This can be viewed as a multifractal decomposition of white noise.)

3. Lévy's uniform modulus of continuity governs the largest increments overall,

$$
\lim _{\varepsilon \rightarrow 0} \sup _{0 \leq t \leq T} \frac{W_{t+\varepsilon}-W_{t}}{(2 \varepsilon|\log \varepsilon|)^{1 / 2}}=1 \quad \text { a.s. }
$$

The three statements above hold in any dimension $d \geq 1$. Next, we indicate their analogues for Brownian occupation measure in dimension $d \geq 3$; only the first of these was previously known.

1'. The limsup asymptotic behavior of Brownian occupation measure around a fixed time $t$ is governed by the LIL of Ciesielski and Taylor [3], Theorem 3; for any $T \in(0, \infty]$ and $t \leq T$,

$$
\limsup _{\varepsilon \rightarrow 0} \frac{\mu_{T}^{W}\left(B\left(W_{t}, \varepsilon\right)\right)}{\varepsilon^{2} \log |\log \varepsilon|}=\frac{2}{q_{d}^{2}} \quad \text { a.s. }
$$

2 . The dimension of exceptional thick times is given by (1.4) above.

$3^{\prime}$. Our results (1.7) and (1.9) give the largest occupation measure possible for a small ball.

\section{Further remarks on Theorem 1.1.}

(i) Perhaps more significant than the numerical values obtained in (1.3) and (1.4) is the insight gained, while proving these results, about the manner by which the "thick points" on the Brownian path arise. The key to our proof of Theorem 1.1 is a localization phenomenon for transient Brownian motion: the balls of radius $\varepsilon$ that have the largest occupation measure (of order $\varepsilon^{2}|\log \varepsilon|$ ), accumulate most of this measure in a surprisingly short time interval (of length at most $\varepsilon^{2}|\log \varepsilon|^{b}$ for some $b$, e.g., $b=6$ works); see Section 3 where this localization is established. The localization phenomenon breaks down in dimension $d=2$, where the correct scaling of occupation measure, and the techniques needed to establish it, are quite different. In [6] we have obtained the corresponding results for the planar case; we emphasize that the current paper concerns only $d \geq 3$.

(ii) Given the localization phenomenon, there are several possible approaches to the proof of the lower bound in (1.4). Our proof relies on a general lower bound on Hausdorff measure of random fractals "of limsup type", Theorem 2.1. This general bound sharpens similar estimates obtained by [13], [9], [4] and [24]; of course, our work owes a substantial debt to these earlier papers. 
(iii) For any $x \notin\left\{W_{t} \mid 0 \leq t \leq T\right\}$ and $\varepsilon$ small enough, $\mu_{T}^{W}(B(x, \varepsilon))=0$. Hence, the equivalence of (1.3) and (1.4) is a direct consequence of the uniform dimension doubling property of Brownian motion, due to Kaufman [10] [see also [18], (0.1)].

(iv) Let $v_{d-2}$ denote the first eigenvalue of $(1 / 2) \Delta$ in the unit ball of $\mathbb{R}^{d-2}$ with Dirichlet boundary conditions. As the spherically symmetric fundamental solution for the Laplacian eigenvalue problem in $B(0,1)$ is $J_{d / 2-2}(\sqrt{\lambda}|x|)$, the required Dirichlet boundary conditions imply that $v_{d-2}=q_{d}^{2} / 2$ [see, e.g., [3], (2.15)]. The appearance of $(d-2)$ in our result for $d$ dimensions is due to the celebrated identity of Ciesielski-Taylor [3], Theorem 2.

To indicate the qualitative difference between the sets of thick points and the most familiar random fractals associated with Brownian motion (the range, the graph, and the level sets) we present the following proposition; for the definition and properties of packing dimension $\operatorname{dim}_{\mathrm{p}}$, see [29] or [8].

Proposition 1.2. Let the notation of Theorem 1.1 be in force. For all $0<a \leq 4 / q_{d}^{2}$, the union Thick ${ }_{\geq a}:=\bigcup_{b \geq a}$ Thick $_{b}$ has the same Hausdorff dimension as Thick $_{a}$ a.s., but its packing dimension a.s. satisfies $\operatorname{dim}_{\mathrm{p}}\left(\right.$ Thick $\left._{\geq a}\right)=1$. Equivalently,

$$
\operatorname{dim}_{\mathrm{P}}\left\{x \in \mathbb{R}^{d} \mid \limsup _{\varepsilon \rightarrow 0} \frac{\mu_{T}^{W}(B(x, \varepsilon))}{\varepsilon^{2}|\log \varepsilon|} \geq a\right\}=2 \quad \text { a.s. }
$$

REMARK. The importance of comparing the Hausdorff and packing dimensions of a set was stressed in Taylor's survey [27]. By a more involved argument, it can be shown that Thick ${ }_{a}$ itself also has packing dimension 1 for $0<a \leq 4 / q_{d}^{2}$. (For $a=4 / q_{d}^{2}$, this statement follows from Proposition 1.2.)

The next theorem solves two problems posed by Taylor in 1974 (see [26], page 201).

THEOREM 1.3. Let $\left\{W_{t}\right\}$ be a Brownian motion in $\mathbb{R}^{d}, d \geq 3$. Then, for any $R \in(0, \infty)$ and any $T \in(0, \infty]$,

$$
\lim _{\varepsilon \rightarrow 0} \sup _{|x| \leq R} \frac{\mu_{T}^{W}(B(x, \varepsilon))}{\varepsilon^{2}|\log \varepsilon|}=4 q_{d}^{-2} \quad \text { a.s. }
$$

Furthermore, for any $k \in(0, \infty)$ and any $T \in[k, \infty]$,

$$
\lim _{\varepsilon \rightarrow 0} \inf _{t \in[0, k]} \frac{\mu_{T}^{W}\left(B\left(W_{t}, \varepsilon\right)\right)}{\varepsilon^{2} /|\log \varepsilon|}=1 \quad \text { a.s. }
$$

REMARKS. Our proof shows that for any $T \in(0, \infty]$,

$$
\lim _{\varepsilon \rightarrow 0} \sup _{0 \leq t \leq T} \frac{\mu_{T}^{W}\left(B\left(W_{t}, \varepsilon\right)\right)}{\varepsilon^{2}|\log \varepsilon|}=4 q_{d}^{-2} \quad \text { a.s. }
$$


Combining (1.3) and (1.7) we see that

$$
\sup _{x \in \mathbb{R}^{d}} \limsup _{\varepsilon \rightarrow 0} \frac{\mu_{\infty}^{W}(B(x, \varepsilon))}{\varepsilon^{2}|\log \varepsilon|}=4 q_{d}^{-2} \quad \text { a.s. }
$$

In particular, the sets in (1.3) and (1.4) are a.s. empty for any $a>4 q_{d}^{-2}$, $T \in(0, \infty]$.

A detailed multifractal analysis of thin times for Brownian motion, that is, $t \in[0, T]$, satisfying

$$
\liminf _{\varepsilon \rightarrow 0} \frac{\mu_{T}^{W}\left(B\left(W_{t}, \varepsilon\right)\right)}{\varepsilon^{2} /|\log \varepsilon|}=a
$$

for some $a \geq 1$, can be found in [7]. The relation between thin times and (1.8) is the same as the relation between thick times and (1.7).

Computation of Laplace transforms is an important component of a complete multifractal analysis, and it was also the starting point of our investigation. Pemantle, Peres and Shapiro [15] showed that $\int_{0}^{1} \mu_{1}^{W}\left(B\left(W_{t}, \varepsilon\right)\right) / \varepsilon^{2} d t$, the pathwise first moment of the ratio $\mu_{1}^{W}\left(B\left(W_{t}, \varepsilon\right)\right) / \varepsilon^{2}$, remains bounded almost surely as $\varepsilon \rightarrow 0$. The following theorem provides a pathwise asymptotic formula for the moment generating function of that ratio. In one sense, it is finer than Theorem 1.1, since it yields a precise estimate of the total duration in $[0,1]$ that the Brownian particle spends in balls of radius $\varepsilon$ that have unusually high occupation measure (see Corollary 1.5 below). Such an estimate (which is an analogue in our setting of the "coarse multifractal spectrum," cf. [22]), cannot be inferred from Theorem 1.1.

THEOREM 1.4. Denote by $\bar{\mu}_{\infty}^{\bar{W}}$ the total occupation measure for a two-sided Brownian motion $\left\{\bar{W}_{t}\right\}_{-\infty}^{\infty}$ in $\mathbb{R}^{d}, d \geq 3$. Then for each $\theta<q_{d}^{2} / 2$,

(1.10) $\lim _{\varepsilon \rightarrow 0} \int_{0}^{1} \exp \left(\theta \mu_{1}^{W}\left(B\left(W_{t}, \varepsilon\right)\right) / \varepsilon^{2}\right) d t=\mathbb{E}\left(\exp \left(\theta \bar{\mu}_{\infty}^{\bar{W}}(B(0,1))\right)\right) \quad$ a.s.

\section{REMARKS.}

(i) We note by [3] that

$$
\begin{aligned}
\mathbb{E}\left(\exp \left(\theta \bar{\mu}_{\infty}^{\bar{W}}(B(0,1))\right)\right) & =\left(\mathbb{E}\left(\exp \left(\theta \mu_{\infty}^{W}(B(0,1))\right)\right)\right)^{2} \\
& =\frac{1}{\prod_{j=1}^{\infty}\left(1-2 \theta / q_{d, j}^{2}\right)^{2}}
\end{aligned}
$$

for each $\theta<q_{d}^{2} / 2$, where $\left\{q_{d, j}\right\}_{j \geq 1}$ are the positive zeros of the Bessel function $J_{d / 2-2}(x)$, enumerated in increasing order. It is clear that the right-hand side diverges as $\theta \uparrow q_{d}^{2} / 2=q_{d, 1}^{2} / 2$. The case $d=3$ is particularly explicit because then $q_{3}=\pi / 2$ and the right-hand side of (1.11) simplifies to $\cos ^{-2}(\sqrt{2 \theta})$ (cf. [3]). 
(ii) Let $\tau$ denote a random variable uniform on $[0,1]$, which is independent of the Brownian path $W$. Then (1.10) implies in particular that for almost every Brownian path $W$, the ratio $\mu_{1}^{W}\left(B\left(W_{\tau}, \varepsilon\right)\right) / \varepsilon^{2}$, a random variable in $\tau$, converges in law as $\varepsilon \rightarrow 0$ to the total occupation time $\bar{\mu}_{\infty}^{\bar{W}}(B(0,1))$ of the unit ball by a two-sided Brownian motion $\bar{W}$.

Next, we state the promised corollary of Theorem 1.4, which is analogous to the coarse multifractal spectrum.

Corollary 1.5. Let $\left\{W_{t}\right\}$ be a Brownian motion in $\mathbb{R}^{d}, d \geq 3$, and denote Lebesgue measure on $\mathbb{R}^{1}$ by $\mathscr{L}$ eb.

Then, for any $a \in\left(0,4 / q_{d}^{2}\right)$,

$$
\lim _{\varepsilon \rightarrow 0} \frac{\log \mathscr{L} e b\left\{0 \leq t \leq 1\left|\mu_{1}^{W}\left(B\left(W_{t}, \varepsilon\right)\right) \geq a \varepsilon^{2}\right| \log \varepsilon \mid\right\}}{\log \varepsilon}=a q_{d}^{2} / 2 \quad \text { a.s. }
$$

The thick points considered in Theorem 1.1 are centers of balls $B(x, \varepsilon)$ with unusually large occupation measure for infinitely many radii, but these radii might be quite rare. The next theorem shows that for the balls $B(x, \varepsilon)$ to have unusually large occupation measure for all small radii $\varepsilon$ and the same center $x$, what constitutes "unusually large" must be interpreted more modestly. Define

$$
I_{d}(a):=\frac{a}{4}\left(\max \left\{0, d-2-\frac{2}{a}\right\}\right)^{2},
$$

and let

$$
C_{d}:=\inf \left\{a: I_{d}(a)=2\right\}=\frac{2}{d-2 \sqrt{d-1}} .
$$

(The equality on the right is easily verified.)

Then we have the following:

THEOREM 1.6. For $\left\{W_{t}\right\}$ a Brownian motion in $\mathbb{R}^{d}, d \geq 3$, and $a \in\left(0, C_{d}\right]$,

$$
\operatorname{dim}\left\{x \in \mathbb{R}^{d} \mid \liminf _{\varepsilon \rightarrow 0} \frac{\mu_{\infty}^{W}(B(x, \varepsilon))}{\varepsilon^{2}} \geq a\right\} \leq 2-I_{d}(a) \text { a.s. }
$$

and this can be strengthened to

$$
\operatorname{dim}_{\mathrm{P}}\left\{x \in \mathbb{R}^{d} \mid \liminf _{\varepsilon \rightarrow 0} \frac{\mu_{\infty}^{W}(B(x, \varepsilon))}{\varepsilon^{2}} \geq a\right\} \leq 2-I_{d}(a) \quad \text { a.s. }
$$

where $\operatorname{dim}_{\mathrm{P}}$ denotes packing dimension. Moreover,

$$
\frac{1}{d} \leq \sup _{x \in \mathbb{R}^{d}} \liminf _{\varepsilon \rightarrow 0} \frac{\mu_{\infty}^{W}(B(x, \varepsilon))}{\varepsilon^{2}} \leq C_{d} \quad \text { a.s. }
$$


REMARKS.

(i) In particular, replacing the limsup by liminf in (1.3) and (1.4) yields an a.s. empty set for any $a>0$.

(ii) The new assertion in (1.16) is the right-hand inequality; the inequality on the left is an immediate consequence of Theorem 9 of Perkins [17] concerning "Brownian slow points."

(iii) It is an open problem to determine exactly the dimension appearing in (1.14) and the precise asymptotics in (1.16).

(iv) That the upper bound (1.14) on Hausdorff dimension applies to packing dimension as well is in sharp contrast with Theorem 1.1 and Proposition 1.2. Intuitively, the reason for this contrast is that for a point to be in the set considered in (1.3), it only needs to satisfy a certain condition at infinitely many scales, so that set can appear large at other scales; these scales can be used to pack many disjoint balls with centers in the set. Points considered in (1.14), however, must satisfy a (less stringent) condition at all scales.

The next section contains a discussion of fractals "of limsup type" and a general lower bound (Theorem 2.1) for their Hausdorff measure. In Section 3 we prove the crucial Localization Lemma 3.1. The results of those two sections are applied in Section 4 to establish the lower bounds on Hausdorff dimension in Theorem 1.1 and Proposition 1.2. The complementary upper bounds in Theorem 1.1 are proved in Section 5. Combining these bounds with the Localization Lemma 3.1, we prove Theorem 1.3 in Section 6. Section 7 is devoted to the proof of Theorem 1.4, with Corollary 1.5 proved in Section 8. Theorem 1.6 is proved in Section 9. At the end of the paper we present some open problems.

Analogous results for transient symmetric stable processes are contained in [5].

2. Random fractals of limsup type. Suppose that for each $n \geq 1$, a finite union $A(n)$ of intervals of length $\lambda_{n}$ is given. Assume that $\lambda_{n} \rightarrow 0$ as $n \rightarrow \infty$ and that the number of intervals comprising $A(n)$ grows like a negative power of $\lambda_{n}$. We call $A:=\lim \sup A(n)=\bigcap_{n=1}^{\infty} \bigcup_{k=n}^{\infty} A(k)$ a fractal of limsup type. We will be interested in situations in which the $A(n)$ are random, and in hypotheses on their distribution which will allow us to obtain dimension bounds on $A$. The main result of this section, Theorem 2.1, provides a general framework for obtaining lower bounds on the Hausdorff measure of random fractals of limsup type.

Random sets that are (well approximated by) random fractals of limsup type include:

1. the fast points of Orey-Taylor [13];

2. the initial points of exceptional Brownian excursions considered in [1];

3. the close approaches on the Brownian path measured by [19];

4. the paths in a family tree where a tree-indexed random walk has positive burst speed; see [2];

5. times where the Strassen functional LIL fails; see [4]; 
6. sets arising in multifractal analysis of stable subordinators (studied in [9] and [24]);

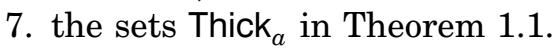

Such random sets differ qualitatively from the random fractals most frequently encountered (e.g., ranges, graphs, levels sets and slow points of Brownian motion). For instance, the packing dimension of sets of limsup type is typically full, hence larger than their Hausdorff dimension; see Corollary 2.4. In particular, that corollary implies that the sets of fast points of [13] have packing dimension 1 (the assertion to the contrary in [27], page 401 is wrong).

Three general methods have been employed to establish lower bounds for Hausdorff dimension of random fractals of limsup type. (These methods were used earlier for other sets).

1. Orey-Taylor [13] constructed a Frostman measure directly, using estimates on binomial probabilities. Their method is expounded in [4]. This elegant method requires strong independence assumptions "within levels," and it is difficult to refine it to handle sets defined by an equality, like Thick ${ }_{a}$, rather than an inequality. Orey-Taylor ([13], page 185) state that this can be done for the random fractals of limsup type, which they consider the Brownian fast points, by "tightening their argument," but extending this to more general situations seems quite hard.

2. Intersection properties with an independent random set (the range of a stable subordinator) were used by [1] and [19]; random Cantor sets arising from fractal percolation as in [16] could also be used. Here independence assumptions can be replaced by correlation bounds, but, as above, handling sets like Thick ${ }_{a}$ is unwieldy.

3. A powerful method based on estimation of energy integrals was used by [9] and [24]. Below we sharpen and extend this method and show that it yields good estimates of Hausdorff measure, while requiring only mild correlation hypothesis.

Let $\mathscr{D}_{n}$ denote the collection of dyadic intervals $\left\{\left[(i-1) 2^{-n}, i 2^{-n}\right]\right\}_{i=1}^{2^{n}}$. For any increasing function $\varphi:[0,1] \rightarrow[0, \infty)$ with $\varphi(0)=0$, let $\mathscr{H}^{\varphi}(A)$ denote the Hausdorff measure of a set $A$ in the gauge $\varphi$ (see, e.g., [27] for the definition).

THEOREM 2.1. Suppose that for every $n \geq 1$, a collection of $\{0,1\}$ valued random variables $\left\{Z_{I}\right\}_{I \in \mathscr{D}_{n}}$ is given, so that $p_{n}:=\mathbf{P}\left(Z_{I}=1\right)$ is the same for all $I \in \mathscr{D}_{n}$. Let

$$
A(n)=\bigcup\left\{I \in \mathscr{D}_{n} \mid Z_{I}=1\right\} \text { and } A:=\lim \sup A(n)=\bigcap_{n=1}^{\infty} \bigcup_{k=n}^{\infty} A(k)
$$

For $I \in \mathscr{D}_{m}$, with $m<n$, define

$$
M_{n}(I)=\sum_{J \in \mathscr{D}_{n}, J \subset I} Z_{J}
$$


Choose $\zeta(n) \geq 1$ such that

$$
\operatorname{Var}\left(M_{n}(I)\right) \leq \zeta(n) \mathbb{E}\left(M_{n}(I)\right)=\zeta(n) p_{n} 2^{n-m} .
$$

Let $\varphi(r)$ be a gauge function which is regularly varying of index $\alpha \in(0,1)$ as $r \downarrow 0$ (i.e., $\varphi(r)=r^{\alpha} L(r)$ where $L(c r) / L(r) \rightarrow 1$ as $r \downarrow 0$ for any $c>0$ ). If

$$
\frac{2^{-n} \zeta(n)}{\varphi\left(2^{-n}\right) p_{n}} \rightarrow 0
$$

then $\mathscr{H}^{\varphi}(A)>0$ a.s.

REMARKS.

(i) We emphasize that no independence or correlation assumptions are made relating $Z_{I}$ and $Z_{J}$ for $I$ and $J$ of different lengths.

(ii) $\mathscr{H}^{\varphi}(A)>0$ immediately implies that $\operatorname{dim}(A) \geq \alpha$.

(iii) Theorem 2.1 can be applied to the "fast points" and "thick points" of a variety of processes; the only essential requirements are stationarity of increments, suitable decay of correlations and (for discontinuous processes) bounds on the jump probabilities. The non-vanishing of Hausdorff measure is proved in Theorem 2.1, rather than merely a bound on dimension, in order to handle the sets Thick ${ }_{a}$, rather than just their unions Thick $_{\geq a}=\bigcup_{b \geq a}$ Thick $_{b}$, in (1.4). (See the remark following the statement of Corollary 4.1.)

(iv) Let $\varphi_{n}:=1 / \varphi\left(2^{-n}\right)$. Beyond the obvious fact of some exponential growth of $\varphi_{n}$, our proof uses only the following simple consequence of the assumption that $\varphi(r)$ is regularly varying of index $\alpha \in(0,1)$ as $r \downarrow 0$ : for some $C<\infty$ that does not depend on $n$,

$$
\sum_{m=1}^{n} \varphi_{m} \leq C \varphi_{n} \quad \text { and } \quad \sum_{m=n}^{\infty} 2^{-m} \varphi_{m} \leq C 2^{-n} \varphi_{n} .
$$

(v) We will apply Theorem 2.1 below to prove Theorem 1.1. In that application, we will take $\varphi(r)=r^{1-\gamma}\left|\log _{2}(r)\right|^{13}$ with $p_{n} \geq 2^{-n \gamma}$ for some $0<\gamma<1$ and $\zeta(n)=n^{12}$, where throughout this paper, $\log _{2}$ stands for the logarithm to the base 2 .

(vi) Theorem 2.1, which is formulated for random fractals of limsup type in $[0,1]$, has an obvious generalization to random "fractals of limsup type" in $[0,1]^{d}$. In this setup we can take $\varphi(r)$ to be any gauge function which is regularly varying of index $\alpha \in(0, d)$ as $r \downarrow 0$, and replace (2.1) and (2.2) by $\operatorname{Var}\left(M_{n}(I)\right) \leq \zeta(n) \mathbb{E}\left(M_{n}(I)\right)=\zeta(n) p_{n} 2^{d(n-m)}$ and $2^{-d n} \zeta(n) /\left(\varphi\left(2^{-n}\right) p_{n}\right) \rightarrow 0$, respectively. The proof of such a generalization is basically identical to the proof of Theorem 2.1.

To establish Theorem 2.1 we need two lemmas. The first one is a version of the well-known connection between energy and Hausdorff measure. For the reader's convenience, we include the brief proof. 
LEMMA 2.2. Fix an increasing gauge function $\varphi$ such that $\varphi(0)=0$. Suppose that $B$ is a Borel set in $[0,1]$, and $\nu$ is a probability measure on $B$. If the dyadic energy

$$
\mathscr{E}_{\varphi}(\nu):=\sum_{m=1}^{\infty} \sum_{J \in \mathscr{D}_{m}} \frac{\nu(J)^{2}}{\varphi\left(2^{-m}\right)}
$$

of $\nu$ is finite, then $\mathscr{H}^{\varphi}(B)>0$.

(In fact $\mathscr{H}^{\varphi}(B)=\infty$, but that is unimportant for our purpose.) See [14] for the connection of $\mathscr{E}_{\varphi}(\nu)$ to more traditional expressions for energy.

ProOF. Let

$$
\Psi(x):=\sum_{m=1}^{\infty} \sum_{J \in \mathscr{D}_{m}} \frac{\nu(J)}{\varphi\left(2^{-m}\right)} \mathbf{1}_{J}(x) .
$$

Since $\int_{B} \Psi(x) d \nu=\mathscr{E}_{\varphi}(\nu)$, taking $C=2 \mathscr{E}_{\varphi}(\nu)$, the set $B_{C}:=\{x \in B \mid \Psi(x) \leq C\}$ has $\nu\left(B_{C}\right) \geq 1 / 2$. The restriction $\nu_{C}$ of $\nu$ to $B_{C}$ satisfies $\nu_{C}(J) \leq C \varphi\left(2^{-m}\right)$ for every $J \in \mathscr{D}_{m}$ for all $m$. Since any interval $I \subset[0,1]$ can be covered by three shorter dyadic intervals, it follows that $\nu_{C}(I) \leq 3 C \varphi(|I|)$ for any interval $I$. Hence, if $\mathscr{A}$ is any countable collection of intervals with $B_{C} \subseteq \bigcup_{\mathscr{A}} I$, then

$$
\frac{1}{2} \leq \nu\left(B_{C}\right) \leq \sum_{\mathscr{A}} \nu_{C}(I) \leq 3 C \sum_{\mathscr{A}} \varphi(|I|)
$$

which implies that $1 /(6 C) \leq \mathscr{H}^{\varphi}\left(B_{C}\right)$.

Alternatively, the a.s. finiteness of $\Psi$, in conjunction with [23], implies that $\mathscr{H}^{\varphi}(B)=\infty$.

The following lemma, which, roughly speaking, controls the "quadratic variation" of the random sets $A(n)$, is the key to the proof of Theorem 2.1. Recall that $\varphi_{n}=1 / \varphi\left(2^{-n}\right)$, and note that by $(2.2)$, for any $l$ we can choose an integer $n(l)>l$ such that

$$
\frac{\varphi_{n(l)} \zeta(n(l))}{2^{n(l)} p_{n(l)}} \leq 2^{-3 l} .
$$

LEMMA 2.3. Let the assumptions of Theorem 2.1 be in force. There exist an a.s. finite random variable $l_{0}(\omega)$ and a constant $C_{3}$, such that if $l \geq l_{0}(\omega)$ and $n=n(l)$, then for all $D \in \mathscr{D}_{l}$, we have

$$
\left|M_{n}(D)-\mathbb{E} M_{n}(D)\right|<\frac{1}{2} \mathbb{E} M_{n}(D)
$$

and

$$
\sum_{m=l}^{n(l)} \varphi_{m} \sum_{J \in \mathscr{D}_{m}, J \subset D} \frac{M_{n}(J)^{2}}{\left(2^{n-l} p_{n}\right)^{2}} \leq C_{3} \varphi_{l}
$$


Proof. For $m \leq n$ and $J \in \mathscr{D}_{m}$, denote

$$
\Delta_{n}(J):=M_{n}(J)-\mathbb{E} M_{n}(J) .
$$

Also, for $l \leq n$ and $D \in \mathscr{D}_{l}$, set

$$
\Upsilon_{n}(D):=\sum_{m=l}^{n} \varphi_{m} \sum_{J \in \mathscr{D}_{m}, J \subset D} \Delta_{n}(J)^{2} .
$$

For $J \in \mathscr{D}_{m}$, the assumption (2.1) gives $\mathbb{E}\left[\Delta_{n}(J)^{2}\right] \leq \zeta(n) p_{n} 2^{n-m}$. Therefore,

$$
\forall D \in \mathscr{D}_{l}, \quad \mathbb{E Y}_{n}(D)=\sum_{m=l}^{n} \varphi_{m} 2^{m-l} \zeta(n) p_{n} 2^{n-m}=2^{n-l} \zeta(n) p_{n} \sum_{m=l}^{n} \varphi_{m} .
$$

By (2.3), we then have

$$
\mathbb{E} Y_{n}(D) \leq C 2^{n-l} p_{n}^{2} \varphi_{n} \zeta(n) / p_{n} .
$$

Thus, by (2.4), since $n=n(l)$,

$$
\mathbb{E} \sum_{D \in \mathscr{O}_{l}} \frac{\Upsilon_{n}(D)}{\left(2^{n-l} p_{n}\right)^{2}} \leq C 2^{-l}
$$

Since the right-hand side is summable in $l$, we conclude that the summands inside the last expectation tend to 0 a.s. as $l \rightarrow \infty$. In particular, there exists $l_{0}(\omega)<\infty$ such that for all $l \geq l_{0}(\omega)$ and $D \in \mathscr{D}_{l}$, we have

$$
\Upsilon_{n}(D) \leq\left(2^{n-l} p_{n}\right)^{2}=\left[\mathbb{E} M_{n}(D)\right]^{2}
$$

To deduce (2.5), observe that

$$
\Delta_{n}(D)^{2} \leq \varphi_{l}^{-1} \Upsilon_{n}(D) \leq \varphi_{l}^{-1}\left[\mathbb{E} M_{n}(D)\right]^{2}<\frac{1}{4}\left[\mathbb{E} M_{n}(D)\right]^{2} .
$$

Next, we calculate

$$
\sum_{J \in \mathscr{D}_{m}, J \subset D} \frac{\left[\mathbb{E} M_{n}(J)\right]^{2}}{\left(2^{n-l} p_{n}\right)^{2}}=\sum_{J \in \mathscr{D}_{m}, J \subset D} 2^{2(l-m)}=2^{l-m} .
$$

Therefore, by (2.3),

$$
\sum_{m=l}^{n} \varphi_{m} \sum_{J \in \mathscr{D}_{m}, J \subset D} \frac{\left[\mathbb{E} M_{n}(J)\right]^{2}}{\left(2^{n-l} p_{n}\right)^{2}}=2^{l} \sum_{m=l}^{n} 2^{-m} \varphi_{m} \leq C \varphi_{l} .
$$

Rewrite (2.7) in the form

$$
\begin{aligned}
\sum_{m=l}^{n} \varphi_{m} & \sum_{J \in \mathscr{D}_{m}, J \subset D} \frac{\Delta_{n}(J)^{2}}{\left(2^{n-l} p_{n}\right)^{2}} \\
= & \frac{1}{\left(2^{n-l} p_{n}\right)^{2}} \sum_{m=l}^{n} \varphi_{m} \sum_{J \in \mathscr{D}_{m}, J \subset D} \Delta_{n}(J)^{2} \leq 1 .
\end{aligned}
$$

Since

$$
M_{n}(J)^{2}=\left[\mathbb{E} M_{n}(J)+\Delta_{n}(J)\right]^{2} \leq 2\left[\mathbb{E} M_{n}(J)\right]^{2}+2 \Delta_{n}(J)^{2},
$$

adding the inequalities (2.8) and (2.9) yields (2.6), for some constant $C_{3}$. 
Proof of Theorem 2.1. We use freely the terminology introduced in the statement of Lemma 2.3. With $l_{0}=l_{0}(\omega)$ as in the lemma, define inductively $l_{k+1}:=n\left(l_{k}\right)$ for $k \geq 0$. For $D \in \mathscr{D}_{l_{k-1}}$ with $k \geq 1$, write

$$
Q_{k}:=\mathbb{E} M_{l_{k}}(D)=2^{l_{k}-l_{k-1}} p_{l_{k}},
$$

and note that by (2.5),

$$
\forall k \geq 1 \forall D \in \mathscr{D}_{l_{k-1}}, \quad \frac{1}{2} Q_{k} \leq M_{l_{k}}(D) \leq 2 Q_{k} .
$$

Summing this over $D \in \mathscr{D}_{l_{k-1}}$ gives

$$
\forall k \geq 1, \quad M_{l_{k}}([0,1]) \leq 2^{l_{k-1}+1} Q_{k} .
$$

To establish the theorem, we will construct a (random) probability measure $\nu$, supported on $\bigcap_{k \geq 1} A\left(l_{k}\right) \subset A$, such that $\mathscr{E}_{\varphi}(\nu)<\infty$ a.s. To specify $\nu$, it suffices to define $\nu(J)$ consistently for all binary intervals $J$. Start by assigning the leftmost interval in $\mathscr{D}_{l_{0}}$ full measure, that is, set $\nu\left[0,2^{-l_{0}}\right]:=1$. Continue inductively: if $J \in \mathscr{D}_{m}$ with $l_{k-1}<m \leq l_{k}$ and $J \subset D$ with $D \in \mathscr{D}_{l_{k-1}}$, define

$$
\nu(J):=\frac{M_{l_{k}}(J) \nu(D)}{M_{l_{k}}(D)} .
$$

It is straightforward to verify that this assignment is consistent and that $\nu$ is supported on $\bigcap_{k \geq 1} A\left(l_{k}\right)$. For $k \geq 2$ and $J$ as in (2.12), two applications of (2.10) and the bound

$$
\nu(D) \leq \frac{Z_{D}}{\min _{\tilde{D} \in \mathscr{I}_{l_{k-2}}} M_{l_{k-1}}(\tilde{D})}
$$

give

$$
\nu(J) \leq \frac{2 M_{l_{k}}(J) \nu(D)}{Q_{k}} \leq \frac{4 M_{l_{k}}(J) Z_{D}}{Q_{k} Q_{k-1}} .
$$

Now we apply Lemma 2.3. For $k \geq 2$ and $D \in \mathscr{D}_{l_{k-1}}$,

$$
\begin{aligned}
& \sum_{m=l_{k-1}}^{l_{k}} \varphi_{m} \sum_{J \in \mathscr{O}_{m}, J \subset D} \nu(J)^{2} \\
& \quad \leq \frac{16 Z_{D}}{Q_{k-1}^{2}} \sum_{m=l_{k-1}}^{l_{k}} \varphi_{m} \sum_{J \in \mathscr{I}_{m}, J \subset D} \frac{M_{l_{k}}(J)^{2}}{Q_{k}^{2}} \leq \frac{16 C_{3} Z_{D}}{Q_{k-1}^{2}} \varphi_{l_{k-1}},
\end{aligned}
$$

by the definition of $Q_{k}$ and (2.6). Summing this over all $D \in \mathscr{D}_{l_{k-1}}$, and then using (2.11) with $k-1$ in place of $k$, we obtain

$$
\begin{aligned}
\sum_{m=l_{k-1}}^{l_{k}} \varphi_{m} \sum_{J \in \mathscr{I}_{m}} \nu(J)^{2} & \leq \frac{16 C_{3} M_{l_{k-1}}([0,1])}{Q_{k-1}^{2}} \varphi_{l_{k-1}} \leq \frac{C_{4} 2^{l_{k-2}}}{Q_{k-1}} \varphi_{l_{k-1}} \\
& \leq \frac{C_{4} 2^{2 l_{k-2}}}{2^{l_{k-1}} p_{l_{k-1}}} \varphi_{l_{k-1}} \leq C_{4} 2^{-l_{k-2}}
\end{aligned}
$$


where the last step used (2.4) and the fact that $\zeta \geq 1$. As the right-hand side of (2.15) is summable in $k$, we conclude that

$$
\mathscr{E}_{\varphi}(\nu)=\sum_{m=0}^{\infty} \varphi_{m} \sum_{J \in \mathscr{D}_{m}} \nu(J)^{2}<\infty \text { a.s. }
$$

By Lemma 2.2, this completes the proof.

The next corollary will be used to prove Proposition 1.2 in Section 4. For $K \subset[0,1]$, let $\mathscr{N}_{m}(K)$ denote the number of intervals in $\mathscr{D}_{m}$ that intersect $K$. Denote by

$$
\overline{\operatorname{dim}}_{\mathrm{M}}(K):=\limsup _{m \rightarrow \infty} \frac{\log _{2} \mathscr{N}_{m}(K)}{m} .
$$

the upper Minkowski dimension of $K$, also known as the upper box dimension.

The only property of packing dimension $\operatorname{dim}_{\mathrm{p}}$ that we need is its relation to Minkowski dimension: for any Borel set $E$,

$$
\operatorname{dim}_{\mathrm{P}}(E)=\inf _{E \subset \cup_{j} E_{j}} \sup _{j} \overline{\operatorname{dim}}_{\mathrm{M}}\left(E_{j}\right),
$$

where the infimum is over all countable covers $\left\{E_{j}\right\}$ of $E$ by closed sets. See [30] or [8], Proposition 3.8.

COROLLARY 2.4. (i) If for each $n \geq 1$ the set $V_{n}$ is relatively open and dense in $[0,1]^{d}$, then $\operatorname{dim}_{\mathrm{p}}\left(\cap_{n} V_{n}\right)=d$.

(ii) In the setting of Theorem 2.1 , the random set $A=\lim \sup A(n)$ satisfies $\operatorname{dim}_{\mathrm{p}}(A)=1$ a.s.

Proof. (i) Let $\left\{E_{j}\right\}$ be a countable collection of closed sets that cover $\bigcap_{n} V_{n}$. Then the union $\left\{E_{j}\right\}_{j \geq 1} \cup\left\{V_{n \geq 1}^{c}\right\}_{n \geq 1}$ is a countable cover of [0,1] $]^{d}$ consisting of closed sets. By Baire's theorem, at least one of these closed sets must have nonempty interior in $[0,1]^{d}$; since each $V_{n}$ is dense, some $E_{j}$ must have interior. From (2.17), we conclude that $\operatorname{dim}_{\mathrm{p}}\left(\cap_{n} V_{n}\right)=d$.

(ii) Denote by $A(n)^{\circ}$ the interior of $A(n)$, so that $V_{n}:=\bigcup_{k=n}^{\infty} A(k)^{\circ}$ is certainly open in $[0,1]$. Fixing a dyadic interval $I$ in $[0,1]$ it is easy to check that Theorem 2.1 applies also when $\mathscr{D}_{n}$ are the dyadic subintervals of $I$. Hence, a.s., for each dyadic $I$, the set $A \cap I$, of positive Hausdorff dimension, is uncountable. Since $A \backslash V_{n}$ is countable, each $V_{n}$ is a.s. dense in [0,1], so the assertion follows from (i).

3. Localization. Throughout this section, $c, c^{\prime}$ denote positive, finite constants, independent of $\varepsilon$, the values of which may change from line to line, using the notation $a \sim b$ if $\lim _{\varepsilon \rightarrow 0} a / b=1$.

To derive lower bounds on the Hausdorff dimension of the sets appearing in Theorem 1.1, as well as for proving (1.7) in Theorem 1.3, it is crucial to be able to consider the occupation measure of a ball of radius $\varepsilon$ over a small time 
interval (of length $\delta_{\varepsilon}$ which tends to zero with $\varepsilon$ ), rather than over an interval of constant length.

Surprisingly, it turns out that with only a small loss in probability, we can work with rather short time intervals; the following lemma makes this precise.

LEMMA 3.1 (Localization lemma). Let $\left\{W_{t}\right\}$ be a Brownian motion in $\mathbb{R}^{d}$, $d \geq 3$. Write $h(r):=r^{2}|\log r|$, and $\theta^{*}:=\Lambda_{d}^{-1}=q_{d}^{2} / 2$. Finally, denote $\delta_{\varepsilon}:=$ $\varepsilon^{2}|\log \varepsilon|^{6}$ and $\beta_{\varepsilon}:=1-2|\log \varepsilon|^{-2}$. Then for some $0<c<\infty$, we have

$$
\mathbf{p}_{\varepsilon}:=\mathbf{P}\left(\mu_{\delta_{\varepsilon}}^{W}\left(B\left(0, \varepsilon \beta_{\varepsilon}\right)\right) \geq a h(\varepsilon)\right) \geq c \varepsilon^{a \theta^{*}} .
$$

We did not attempt to optimize the powers of $|\log \varepsilon|$ appearing in the definitions of $\delta_{\varepsilon}$ and $\beta_{\varepsilon}$. Nevertheless, to appreciate the sharpness of this lemma, recall that by [3] [cf. (3.4) below],

$$
\mathbf{P}\left(\mu_{\infty}^{W}(B(0, \varepsilon)) \geq a h(\varepsilon)\right) \sim c^{\prime} \varepsilon^{a \theta^{*}} .
$$

Proof. Define

$$
\mathscr{T}=\mathscr{T}(\varepsilon):=\inf \left\{s \geq 0:\left|W_{s}\right|=\varepsilon|\log \varepsilon|^{2}\right\} .
$$

By Brownian scaling, we deduce the existence of positive constants $c_{1}, c_{2}$ such that

$$
\mathbf{P}\left(\mathscr{T}>\delta_{\varepsilon}\right)=\mathbf{P}\left(\sup _{t \in\left[0,|\log \varepsilon|^{2}\right]}\left|W_{t}\right| \leq 1\right) \sim c_{1} \exp \left(-c_{2}|\log \varepsilon|^{2}\right) .
$$

Therefore,

$$
\begin{aligned}
\mathbf{p}_{\varepsilon} & \geq \mathbf{P}\left(\varepsilon^{-2} \int_{0}^{\mathscr{T}} \mathbf{1}_{\left\{\left|W_{s}\right|<\varepsilon \beta_{\varepsilon}\right\}} d s \geq a|\log \varepsilon| ; \mathscr{T} \leq \delta_{\varepsilon}\right) \\
& \geq \mathbf{P}\left(\varepsilon^{-2} \int_{0}^{\mathscr{T}} \mathbf{1}_{\left\{\left|W_{s}\right|<\varepsilon \beta_{\varepsilon}\right\}} d s \geq a|\log \varepsilon|\right)-\mathbf{P}\left(\mathscr{T}>\delta_{\varepsilon}\right) .
\end{aligned}
$$

By (3.1) and (3.2), the lemma will be proved once we establish that

$$
\mathbf{P}\left(\varepsilon^{-2} \int_{0}^{\mathscr{T}} \mathbf{1}_{\left\{\left|W_{s}\right|<\varepsilon \beta_{\varepsilon}\right\}} d s \geq a|\log \varepsilon|\right) \geq c \varepsilon^{a \theta^{*}} .
$$

To see (3.3), denote by $\tau_{d-2}$ the hitting time of the unit sphere in $\mathbb{R}^{d-2}$ by Brownian motion, and define

$$
\begin{aligned}
I & =\varepsilon^{-2} \int_{0}^{\infty} \mathbf{1}_{\left\{\left|W_{s}\right|<\varepsilon \beta_{\varepsilon}\right\}} d s, \\
I^{\mathscr{T}} & =\varepsilon^{-2} \int_{0}^{\mathscr{T}} \mathbf{1}_{\left\{\left|W_{s}\right|<\varepsilon \beta_{\varepsilon}\right\}} d s .
\end{aligned}
$$


Recall that, using [3] for the first equality,

$$
\frac{\mathbf{P}\left(\int_{0}^{\infty} \mathbf{1}_{\left\{\left|W_{s}\right|<1\right\}} d s \geq x\right)}{e^{-x \theta^{*}}}=\frac{\mathbf{P}\left(\tau_{d-2} \geq x\right)}{e^{-x \theta^{*}}} \rightarrow c \quad \text { as } x \rightarrow \infty .
$$

Therefore, using Brownian scaling and (3.4),

$$
\begin{aligned}
\mathbf{P}(I \geq a|\log \varepsilon|) & =\mathbf{P}\left(\left(\beta_{\varepsilon} \varepsilon\right)^{-2} \int_{0}^{\infty} \mathbf{1}_{\left\{\left|W_{s}\right|<\varepsilon \beta_{\varepsilon}\right\}} d s \geq \beta_{\varepsilon}^{-2} a|\log \varepsilon|\right) \\
& =\mathbf{P}\left(\tau_{d-2} \geq \beta_{\varepsilon}^{-2} a|\log \varepsilon|\right) \\
& \sim c \exp \left(-\theta^{*} a|\log \varepsilon| /\left(1-2|\log \varepsilon|^{-2}\right)^{2}\right) \sim c \varepsilon^{a \theta^{*}} .
\end{aligned}
$$

Let now $\mathscr{T}^{\prime}:=\inf \left\{t>\mathscr{T}:\left|W_{t}\right|<\varepsilon\right\}$, and define

$$
I_{\mathscr{T}^{\prime}}=\varepsilon^{-2} \int_{\mathscr{T}^{\prime}}^{\infty} \mathbf{1}_{\left\{\left|W_{s}\right|<\varepsilon \beta_{\varepsilon}\right\}} d s .
$$

Then, $I=I^{\mathscr{T}} \mathbf{1}_{\left\{\mathscr{T}^{\prime}=\infty\right\}}+\left(I^{\mathscr{T}}+I_{\mathscr{T}^{\prime}}\right) \mathbf{1}_{\left\{\mathscr{T}^{\prime}<\infty\right\}}$ so that

$$
\mathbf{P}\left(I^{\mathscr{T}} \geq z ; \mathscr{T}^{\prime}=\infty\right)=\mathbf{P}(I \geq z)-\mathbf{P}\left(I^{\mathscr{T}}+I_{\mathscr{T}^{\prime}} \geq z ; \mathscr{T}^{\prime}<\infty\right) .
$$

Let $\tilde{I}$ be an independent copy of $I$ for a Brownian motion whose expectation when starting at $v$ we denote by $\tilde{\mathbb{E}}^{v}$. Using symmetry and the strong Markov property we have

$$
\begin{aligned}
\mathbf{P}\left(I^{\mathscr{T}}+I_{\mathscr{T}^{\prime}} \geq z ; \mathscr{T}^{\prime}<\infty\right) & =\mathbb{E}\left(\tilde{\mathbb{E}}^{W_{\mathscr{T}^{\prime}}}\left\{\tilde{I} \geq z-I^{\mathscr{T}}\right\} ; \mathscr{T}^{\prime}<\infty\right) \\
& \leq \mathbb{E}\left(\tilde{\mathbb{E}}\left\{\tilde{I} \geq z-I^{\mathscr{T}}\right\} ; \mathscr{T}^{\prime}<\infty\right) \\
& =\tilde{\mathbb{E}}\left\{\mathbb{E}\left(I^{\mathscr{T}} \geq z-\tilde{I} ; \mathscr{T}^{\prime}<\infty\right)\right\} \\
& =\tilde{\mathbb{E}}\left\{\mathbb{E}\left(\mathbb{E}^{W_{\mathscr{T}}}\left(T_{B(0, \varepsilon)}<\infty\right) ; I^{\mathscr{T}} \geq z-\tilde{I}\right)\right\} \\
& =|\log \varepsilon|^{-2(d-2)} \mathbf{P}\left(I^{\mathscr{T}}+\tilde{I} \geq z\right) \\
& \leq|\log \varepsilon|^{-2(d-2)} \mathbf{P}(I+\tilde{I} \geq z),
\end{aligned}
$$

where $T_{B(0, \varepsilon)}=\inf \left\{t \geq 0: W_{t} \in B(0, \varepsilon)\right\}$ denotes the first hitting time of $B(0, \varepsilon)$.

Let $\tilde{\tau}_{d-2}$ denote an independent copy of $\tau_{d-2}$, and let $q_{\tau}$ denote their common law. Then, for some constant $C$ independent of $z$, which may change from line to line,

$$
\begin{aligned}
\mathbf{P}\left(\tau_{d-2}+\tilde{\tau}_{d-2}>z\right) & =\mathbf{P}\left(\tau_{d-2}>z\right)+\int_{0}^{z} \mathbf{P}\left(\tilde{\tau}_{d-2}>z-y\right) q_{\tau}(d y) \\
& \leq C\left[\exp \left(-z \theta^{*}\right)+\int_{0}^{z} \exp \left(-(z-y) \theta^{*}\right) q_{\tau}(d y)\right] \\
& \leq C \exp \left(-z \theta^{*}\right)+C \int_{0}^{z} \exp \left(-z \theta^{*}\right) d y \\
& =C(1+z) \exp \left(-z \theta^{*}\right)
\end{aligned}
$$


where the third line came from integration by parts. Hence, by the same argument as in (3.5), for some $c>0$ and any $\varepsilon>0$ small enough,

$$
\mathbf{P}(I+\tilde{I} \geq a|\log \varepsilon|) \leq c a|\log \varepsilon| \varepsilon^{a \theta^{*}} .
$$

Since $2(d-2)>1$, the inequality (3.3) follows from (3.5), (3.6), (3.7) and the above.

4. Proof of the lower bound and critical case in Theorem 1.1. The following corollary of Theorem 2.1 and the Localization Lemma will yield the desired lower bound.

Recall that $\theta^{*}=\Lambda_{d}^{-1}=q_{d}^{2} / 2$ denotes the first eigenvalue of the Dirichlet half-Laplacian in the unit ball of $\mathbb{R}^{d-2}$.

Corollary 4.1. Let $T \in(0, \infty]$ and $a \in\left(0,2 \Lambda_{d}\right)$. Denote $h(\varepsilon)=\varepsilon^{2}|\log \varepsilon|$, and consider the set of "thick times,"

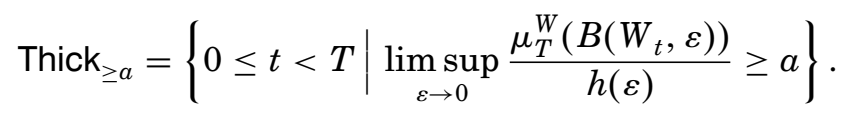

Let $\gamma=a \theta^{*} / 2 \in(0,1)$ and $\varphi(r)=r^{1-\gamma}\left|\log _{2} r\right|^{13}$. Then $\mathscr{H}^{\varphi}\left(\right.$ Thick $\left._{\geq a}\right)>0$ a.s.

4.1. Derivation of the lower bound in Theorem 1.1 Assuming for the moment the upper bounds on dimension obtained in Section 5, we may infer that $\operatorname{dim}\left(\right.$ Thick $\left._{a}\right)=1-\gamma$ as follows (cf. the argument in [13], page 185). The inequality $\operatorname{dim}\left(\right.$ Thick $\left._{\geq(a+1 / n)}\right) \leq 1-(a+1 / n) \theta^{*} / 2$ of Section 5 implies that $\mathscr{H}^{\varphi}\left(\right.$ Thick $\left._{\geq(a+1 / n)}\right)=0$, and since Thick $_{a}=$ Thick $_{\geq a}-\bigcup_{n=1}^{\infty}$ Thick $_{\geq(a+1 / n)}$, Corollary 4.1 shows that $\mathscr{H}^{\varphi}\left(\right.$ Thick $\left._{a}\right)>0$ which in turn implies that $\operatorname{dim}\left(\right.$ Thick $\left._{a}\right) \geq$ $1-\gamma$. Using once again the upper bound from Section 5 then completes the proof that $\operatorname{dim}\left(\right.$ Thick $\left._{a}\right)=1-\gamma$.

4.2. Derivation of the critical case in Theorem 1.1 We now show that Thick $_{4 / q_{d}^{2}} \neq \varnothing$; perhaps surprisingly, this can be done by a "soft" argument. For $h>0$ and $a<4 / q_{d}^{2}$, consider the set of approximate thick times

$$
\operatorname{Thick}(a, h):=\bigcup_{\varepsilon \in(0, h)}\left\{0<t<T \mid \frac{\mu_{T}^{W}\left(B\left(W_{t}, \varepsilon\right)\right)}{\varepsilon^{2}|\log \varepsilon|}>a\right\} .
$$

For any $a<4 / q_{d}^{2}$ and $h>0$, it follows from (1.4) and the Markov property of Brownian motion that Thick $(a, h)$ is a.s. dense in $[0, T]$, and it is easy to check that Thick $(a, h)$ is an open set. Thus fixing sequences $a_{n} \uparrow 4 / q_{d}^{2}$ and $h_{n} \downarrow 0$, Baire's category theorem implies that

$$
\bigcap_{n} \operatorname{Thick}\left(a_{n}, h_{n}\right) \neq \varnothing \text {. }
$$

Finally, inspection shows that this intersection coincides with Thick $\mathrm{K}_{\geq 4 / q_{d}^{2}}$, which in turn coincides with Thick $_{4 / q_{d}^{2}}$ by the remark following Theorem 1.3. 
ProOF OF COROLlary 4.1. Since we are proving a lower bound, we may assume that $T$ is finite; by Brownian scaling, it is enough to consider $T=2$. Take $\varepsilon_{n}=n^{3} 2^{-n / 2}, n=1,2, \ldots$ and $\beta_{\varepsilon_{n}}=1-2\left|\log \varepsilon_{n}\right|^{-2}$ as in the Localization Lemma. With $I=\left[t, t+2^{-n}\right] \in \mathscr{D}_{n}$, define $\tilde{I}=\left[t, t+n^{12} 2^{-n}\right]$, and let

$$
Z_{I}=1 \text { iff } \int_{\tilde{I}} \mathbf{1}_{\left\{\left|W_{s}-W_{t}\right|<\varepsilon_{n} \beta_{\varepsilon_{n}}\right\}} d s \geq a h\left(\varepsilon_{n}\right) .
$$

By Lévy's uniform modulus of continuity, there exists an a.s. finite random variable $n_{0}(\omega)$, such that, for all $n \geq n_{0}(\omega)$,

$$
\sup \left\{\left|W_{t}-W_{t^{\prime}}\right|: t, t^{\prime} \in[0,1],\left|t-t^{\prime}\right| \leq 2^{-n}\right\} \leq 2 \sqrt{2^{-n} \log \left(2^{n}\right)} .
$$

Therefore, for all $n>n_{0}(\omega)$, if $I \in \mathscr{D}_{n}$ and $Z_{I}=1$, then

$$
\int_{\tilde{I}} \mathbf{1}_{\left\{\left|W_{s}-W_{t^{\prime}}\right|<\varepsilon_{n}\right\}} d s \geq a h\left(\varepsilon_{n}\right)
$$

for every $t^{\prime} \in I$. The set $A$ defined in Theorem 2.1 satisfies $A \subset$ Thick $_{>a}$ a.s. (we have taken $T=2$ rather than $T=1$ to avoid boundary effects here). The localization lemma (Lemma 3.1) shows that for $I \in \mathscr{D}_{n}$, and all $n$ large enough, $p_{n}=\mathbf{P}\left(Z_{I}=1\right) \geq 2^{-a \theta^{*} n / 2}$. Thus, Corollary 4.1 will be established once we verify the variance condition (2.1). For intervals $I, J \in \mathscr{D}_{n}$ the variables $Z_{I}$ and $Z_{J}$ always satisfy $\operatorname{Cov}\left(Z_{I}, Z_{J}\right) \leq \mathbb{E}\left(Z_{I}\right)=p_{n}$, and $\operatorname{if} \operatorname{dist}(I, J)>n^{12} 2^{-n}$, then $Z_{I}$ and $Z_{J}$ are independent. Therefore, fixing $m<n$ and $D \in \mathscr{D}_{m}$, each $I \in \mathscr{D}_{n}$ satisfies $\operatorname{Cov}\left(Z_{I}, M_{n}(D)\right) \leq n^{12} p_{n}$. Consequently

$$
\operatorname{Var}\left(M_{n}(D)\right)=\sum_{I \in \mathscr{D}_{n}, I \subset D} \operatorname{Cov}\left(Z_{I}, M_{n}(D)\right) \leq 2^{n-m} n^{12} p_{n}
$$

Hence, Theorem 2.1 may be applied (with $p_{n} \geq 2^{-\gamma n}$ and $\zeta(n)=n^{12}$ ) to yield the conclusion.

Proof of Proposition 1.2. In the course of the proof of Corollary 4.1, we showed that for $a \in\left(0,2 \Lambda_{d}\right)$, the set Thick ${ }_{\geq a}$ contains a set of the form $A=$ $\lim \sup A(n)$ that satisfies the hypotheses of Theorem 2.1. Thus, the assertion $\operatorname{dim}_{\mathrm{p}}\left(\right.$ Thick $\left._{\geq a}\right)=1$ follows immediately from Corollary 2.4(ii). To handle the critical case $a=4 / q_{d}^{2}=2 \Lambda_{d}$, observe that in the analysis of that case earlier in this section, we expressed Thick ${ }_{\geq a}$ as a countable intersection of dense open sets, so $\operatorname{dim}_{\mathrm{p}}\left(\right.$ Thick $\left._{\geq a}\right)=1$ by Corollary 2.4(i). (This method also applies to smaller $a$.) Finally, we may deduce (1.6) from the uniform doubling of packing dimension by spatial Brownian motion, established in [18], Corollary 5.8.

5. The upper bound in Theorem 1.1. In this section we establish the upper bound for (1.3), thus completing the proof of Theorem 1.1. Let $\left\{W_{t}\right\}_{t \geq 0}$ be a Brownian motion in $\mathbb{R}^{d}, d \geq 3$, and $h(\varepsilon)=\varepsilon^{2}|\log \varepsilon|$. Set

$$
z_{T}(x, \varepsilon):=\mu_{T}^{W}(B(x, \varepsilon)) / h(\varepsilon),
$$


with $z(x, \varepsilon)=z_{\infty}(x, \varepsilon)$. In this section we show that

$$
\operatorname{dim}\left\{x \in B(0, k) \mid \limsup _{\varepsilon \rightarrow 0} z(x, \varepsilon) \geq a\right\} \leq 2-a \Lambda_{d}^{-1}
$$

a.s. for all $a \leq 2 \Lambda_{d}, k \in[1, \infty)$. Using $z(x, \varepsilon) \geq z_{T}(x, \varepsilon)$, and considering the countable union over $k=1,2, \ldots$, will then complete the proof of the upper bound on the dimension of sets in (1.3).

Fix $k \in[1, \infty)$ and $\delta \in(0,1 / 5)$. Choose a sequence $\varepsilon_{n} \downarrow 0$ as $n \rightarrow \infty$ in such a way that $\varepsilon_{1}<e^{-2}$ and

$$
h\left(\varepsilon_{n+1}\right)=(1-\delta) h\left(\varepsilon_{n}\right)
$$

For any $a>0$ let

$$
D_{a}:=\left\{x \in B(0, k) \mid \limsup _{n \rightarrow \infty} z\left(x, \varepsilon_{n}\right) \geq(1-\delta) a\right\} .
$$

Since, for $\varepsilon_{n+1} \leq \varepsilon \leq \varepsilon_{n}$ we have

$$
z\left(x, \varepsilon_{n}\right)=\frac{h\left(\varepsilon_{n+1}\right)}{h\left(\varepsilon_{n}\right)} \frac{\mu_{\infty}^{W}\left(B\left(x, \varepsilon_{n}\right)\right)}{h\left(\varepsilon_{n+1}\right)} \geq(1-\delta) z(x, \varepsilon),
$$

it is easy to see that

$$
\left\{x \in B(0, k) \mid \limsup _{\varepsilon \rightarrow 0} z(x, \varepsilon) \geq a\right\} \subseteq D_{a} .
$$

Let $\left\{x_{j}: j=0,1, \ldots, K_{n}\right\}$, with $x_{0}=0$, denote a maximal collection of points in $B(0, k)$ such that $\inf _{l \neq j}\left|x_{l}-x_{j}\right| \geq \delta \varepsilon_{n}$. Let $\mathscr{A}_{n}$ be the set of $j, 0 \leq j \leq K_{n}$, such that

$$
\mu_{\infty}^{W}\left(B\left(x_{j},(1+\delta) \varepsilon_{n}\right)\right) \geq(1-2 \delta) a h\left(\varepsilon_{n}\right) .
$$

We will shortly prove that for any $a>0$,

$$
\mathbb{E}\left|\mathscr{A}_{n}\right| \leq c^{\prime} \varepsilon_{n}^{(1-4 \delta) a \theta^{*}-2} \text {. }
$$

Assuming this for the moment, fix $a \leq 2 / \theta^{*}$ and let $\mathscr{V}_{n, j}=B\left(x_{j}, \delta \varepsilon_{n}\right)$. For any $x \in B(0, k)$ there exists $j \in\left\{0, \ldots, K_{n}\right\}$ such that $x \in \mathscr{V}_{n, j}$ and $B\left(x, \varepsilon_{n}\right) \subseteq B\left(x_{j},(1+\delta) \varepsilon_{n}\right)$. Consequently, $\bigcup_{n \geq m} \bigcup_{j \in \mathscr{A}_{n}} \mathscr{V}_{n, j}$ forms a cover of $D_{a}$ by sets of maximal diameter $2 \delta \varepsilon_{m}$. Since $\mathscr{V}_{n, j}$ have diameter $2 \delta \varepsilon_{n}$, it follows from (5.4) that for $\gamma=2-(1-5 \delta) a \theta^{*}>0$,

$$
\mathbb{E} \sum_{n=m}^{\infty} \sum_{j \in \mathscr{A}_{n}}\left|\mathscr{V}_{n, j}\right|^{\gamma} \leq c^{\prime}(2 \delta)^{\gamma} \sum_{n=m}^{\infty} \varepsilon_{n}^{\delta a \theta^{*}}<\infty .
$$

Thus, $\sum_{n=m}^{\infty} \sum_{j \in \mathscr{A}_{n}}\left|\mathscr{V}_{n, j}\right|^{\gamma}$ is finite a.s. implying that $\operatorname{dim}\left(D_{a}\right) \leq \gamma$ a.s. Taking $\delta \downarrow 0$ completes the proof of the upper bound (5.1), subject only to (5.4) which we now prove. 
Let $\sigma_{j}=\inf \left\{t \geq 0: W_{t} \in B\left(x_{j},(1+\delta) \varepsilon_{n}\right)\right\}$. By the strong Markov property and [3] [cf. (3.4)], for some $c=c(\delta, a, d)<\infty$ and all $n$,

$$
\begin{aligned}
\mathbf{P}\left(\mu_{\infty}^{W}\right. & \left.\left(B\left(x_{j},(1+\delta) \varepsilon_{n}\right)\right) \geq(1-2 \delta) a h\left(\varepsilon_{n}\right)\right) \\
= & \mathbf{P}\left(\mathbb{E}^{W_{\sigma_{j}}-x_{j}}\left(\mu_{\infty}^{W}\left(B\left(0,(1+\delta) \varepsilon_{n}\right)\right) \geq(1-2 \delta) a h\left(\varepsilon_{n}\right)\right) ; \sigma_{j}<\infty\right) \\
& \leq \mathbf{P}\left(\mathbb{E}\left(\mu_{\infty}^{W}\left(B\left(0,(1+\delta) \varepsilon_{n}\right)\right) \geq(1-2 \delta) a h\left(\varepsilon_{n}\right)\right) ; \sigma_{j}<\infty\right) \\
& \leq c \varepsilon_{n}^{(1-4 \delta) a \theta^{*}} \mathbf{P}\left(\sigma_{j}<\infty\right),
\end{aligned}
$$

where the first inequality is due to symmetry. Recall that

$$
\mathbf{P}\left(\sigma_{j}<\infty\right)=\left(\frac{(1+\delta) \varepsilon_{n}}{\left|x_{j}\right|}\right)^{d-2} \wedge 1 .
$$

Hence, for some $c_{1}=c_{1}(\delta, a, d), c^{\prime}=c^{\prime}(\delta, a, d, k)<\infty$ and every $n$,

$$
\begin{aligned}
\mathbb{E}\left|\mathscr{A}_{n}\right| & =\sum_{j=0}^{K_{n}} \mathbf{P}\left(\mu_{\infty}^{W}\left(B\left(x_{j},(1+\delta) \varepsilon_{n}\right)\right) \geq(1-2 \delta) a h\left(\varepsilon_{n}\right)\right) \\
& \leq c_{1} \varepsilon_{n}^{(1-4 \delta) a \theta^{*}-2}\left(1+\int_{|x| \leq k} \frac{1}{|x|^{d-2}} d x\right) \leq c^{\prime} \varepsilon_{n}^{(1-4 \delta) a \theta^{*}-2},
\end{aligned}
$$

which completes the proof of (5.4) and consequently that of Theorem 1.1.

\section{A solution of Taylor's 1974 problems.}

Proof of Theorem 1.3. We begin by proving (1.7). To this end, fix $T \in$ $(0, \infty), \delta \in(0,1 / 4)$ and $a<2 \Lambda_{d}=2 / \theta^{*}$ such that $\eta=2-(1+\delta) a \theta^{*}>0$. Choose a sequence $\varepsilon_{n} \downarrow 0$ as in (5.2), noting that for $\varepsilon_{n} \leq \varepsilon \leq \varepsilon_{n-1}$ and any $x \in \mathbb{R}^{d}$,

$$
(1-\delta) z_{T}\left(x, \varepsilon_{n}\right) \leq z_{T}(x, \varepsilon) \leq(1-\delta)^{-1} z_{T}\left(x, \varepsilon_{n-1}\right) .
$$

Let $\delta_{\varepsilon}=\varepsilon^{2}|\log \varepsilon|^{6}, N_{n}=\left[T / \delta_{\varepsilon_{n}}\right]$, and $t_{i, n}=i \delta_{\varepsilon_{n}}$ for $i=0, \ldots, N_{n}-1$. Writing $W_{s}^{t}=W_{s+t}-W_{t}$, it follows that

$$
\inf _{\varepsilon \in\left[\varepsilon_{n}, \varepsilon_{n-1}\right]} \sup _{t \in[0, T]} z_{T}\left(W_{t}, \varepsilon\right) \geq(1-\delta) \max _{i=0}^{N_{n}-1} Z_{i}^{(n)}
$$

where $Z_{i}^{(n)}=\mu_{\delta_{\varepsilon_{n}}}^{W_{i, n}^{t_{i, n}}}\left(B\left(0, \varepsilon_{n}\right)\right) / h\left(\varepsilon_{n}\right)$ are i.i.d. and by Lemma 3.1, for some $c=c(T)>0$ and all $n$ large enough,

$$
\mathbf{P}\left(\max _{i=0}^{N_{n}-1} Z_{i}^{(n)} \leq a\right) \leq\left(1-\mathbf{p}_{\varepsilon_{n}}\right)^{N_{n}} \leq \exp \left(-c \varepsilon_{n}^{-\eta}\right) .
$$

Applying Borel-Cantelli, then taking $\delta \downarrow 0$ and $a \uparrow 2 \Lambda_{d}$, we see that a.s.,

$$
\liminf _{\varepsilon \rightarrow 0} \sup _{t \in[0, T]} z_{T}\left(W_{t}, \varepsilon\right) \geq 2 \Lambda_{d}=4 q_{d}^{-2} .
$$


With $S_{k}(\omega)=\inf \left\{t:\left|W_{t}\right| \geq k\right\} \wedge T \in(0, \infty)$ a.s. and $T \mapsto z_{T}(x, r)$ monotone nondecreasing, it follows that a.s.,

$$
\liminf _{\varepsilon \rightarrow 0} \sup _{|x| \leq k} z_{T}(x, \varepsilon) \geq \liminf _{\varepsilon \rightarrow 0} \sup _{t \in\left[0, S_{k}(\omega)\right]} z_{S_{k}(\omega)}\left(W_{t}, \varepsilon\right) \geq 4 q_{d}^{-2} .
$$

Turning to the proof of the corresponding upper bound, fix $k \in(0, \infty)$, $\delta \in(0,1 / 5)$ and let $a=(2+\delta) /\left((1-4 \delta) \theta^{*}\right)>2 / \theta^{*}$. Considering the sequence $\varepsilon_{n}$ of (5.2) and the sets $\mathscr{A}_{n}$ as in Section 5, it follows from (5.4) that

$$
\sum_{n=1}^{\infty} \mathbf{P}\left(\left|\mathscr{A}_{n}\right| \geq 1\right) \leq \sum_{n=1}^{\infty} \mathbb{E}\left|\mathscr{A}_{n}\right| \leq c^{\prime} \sum_{n=1}^{\infty} \varepsilon_{n}^{\delta}<\infty .
$$

By Borel-Cantelli, it thus follows that a.s. $\mathscr{A}_{n}$ is empty for all $n \geq n_{0}(\omega)$. By the construction of Section 5 the latter event implies that

$$
\limsup _{\varepsilon \rightarrow 0} \sup _{|x| \leq k} z_{\infty}(x, \varepsilon) \leq a
$$

Taking $\delta \downarrow 0$ for which $a \downarrow 2 / \theta^{*}=4 q_{d}^{-2}$, we conclude that a.s.,

$$
\limsup _{\varepsilon \rightarrow 0} \sup _{|x| \leq k} z_{\infty}(x, \varepsilon) \leq 4 q_{d}^{-2}
$$

as needed to complete the proof of (1.7).

The left side of (1.8) is monotone in $T$ and by Brownian scaling its law depends only on $T / k$. Therefore, it suffices to consider $k=1$ and the extreme values $T=1$ and $T=\infty$. Fix $\delta>0$ and $\varepsilon_{n}=(1-\delta)^{n}$. (Note: this is different from the $\varepsilon_{n}$ used above.) Using the notation

$$
\hat{z}_{T}(x, \varepsilon):=\frac{\mu_{T}^{W}(B(x, \varepsilon))}{\left(\varepsilon^{2} /|\log \varepsilon|\right)},
$$

it follows that for any $\varepsilon \in\left[\varepsilon_{n}, \varepsilon_{n-1}\right]$ and $x \in \mathbb{R}^{d}$,

$$
\frac{n-1}{n}(1-\delta)^{2} \hat{z}_{T}\left(x, \varepsilon_{n}\right) \leq \hat{z}_{T}(x, \varepsilon) \leq \frac{n}{n-1}(1-\delta)^{-2} \hat{z}_{T}\left(x, \varepsilon_{n-1}\right) .
$$

Thus, it suffices for (1.8) to show that for any fixed $\delta \in(0,1 / 5)$ both the lower bound

$$
\liminf _{n \rightarrow \infty} \inf _{t \in[0,1]} \hat{z}_{1}\left(W_{t}, \varepsilon_{n}\right) \geq(1-\delta)^{5}
$$

and the upper bound

$$
\limsup _{n \rightarrow \infty} \inf _{t \in[0,1]} \hat{z}_{\infty}\left(W_{t}, \varepsilon_{n}\right) \leq(1+\delta)^{5},
$$

hold a.s.

Our first task in proving (6.2) is to get a good upper bound on the probability of small occupation measure. If $\mu_{[-a, b]}^{\bar{W}}\left(B\left(0, \varepsilon_{n}\right)\right)$ denotes the occupation measure of a two-sided $\mathbb{R}^{d}$-valued Brownian motion $\bar{W}$ in $B\left(0, \varepsilon_{n}\right)$ during the 
time interval $[-a, b]$ with $a, b \geq 0$, then $\mu_{[-a, b]}^{\bar{W}}\left(B\left(0, \varepsilon_{n}\right)\right) \leq \gamma$ implies that $\bar{\tau}_{d}\left(\varepsilon_{n}\right) \wedge a+\tau_{d}\left(\varepsilon_{n}\right) \wedge b \leq \gamma$, where

$$
\bar{\tau}_{d}(\varepsilon)=\inf \left\{t \geq 0:\left|\bar{W}_{-t}\right| \geq \varepsilon\right\}, \quad \tau_{d}(\varepsilon)=\inf \left\{t \geq 0:\left|\bar{W}_{t}\right| \geq \varepsilon\right\} .
$$

Taking $\gamma=(1-\delta)^{2} \varepsilon_{n}^{2} /\left|\log \varepsilon_{n}\right|$, then $a \wedge b \geq(1-\delta)^{2} \varepsilon_{n}^{2} /\left|\log \varepsilon_{n}\right|$ together with Brownian scaling shows that

$$
\begin{aligned}
& \mathbf{P}\left(\mu_{[-a, b]}^{\bar{W}}\left(B\left(0, \varepsilon_{n}\right)\right) \leq(1-\delta)^{2} \varepsilon_{n}^{2} /\left|\log \varepsilon_{n}\right|\right) \\
& \quad \leq \mathbf{P}\left(\bar{\tau}_{d}(1)+\tau_{d}(1) \leq(1-\delta)^{2} /\left|\log \varepsilon_{n}\right|\right) .
\end{aligned}
$$

Since $\mathbf{P}\left(\tau_{d}(1) \leq x\right)=\mathbf{P}\left(\sup _{0 \leq t \leq x}\left|W_{t}\right| \geq 1\right)$, it is well known (see [29], Lemma 6.4) that for $0<x<1$,

$$
c_{1} x^{1-d / 2} e^{-0.5 / x} \leq \mathbf{P}\left(\tau_{d}(1) \leq x\right) \leq c_{2} x^{1-d / 2} e^{-0.5 / x} .
$$

This estimate leads, as in the proof of [29], Lemma 6.5, to

$$
\mathbf{P}\left(\bar{\tau}_{d}(1)+\tau_{d}(1) \leq x\right) \leq \exp (-2(1-\delta) / x)
$$

for any $\delta>0$ and $x \leq x(\delta)$. Hence, whenever $a \wedge b \geq(1-\delta)^{2} \varepsilon_{n}^{2} /\left|\log \varepsilon_{n}\right|$,

$$
\mathbf{P}\left(\mu_{[-a, b]}^{\bar{W}}\left(B\left(0, \varepsilon_{n}\right)\right) \leq(1-\delta)^{2} \varepsilon_{n}^{2} /\left|\log \varepsilon_{n}\right|\right) \leq \varepsilon_{n}^{2 /(1-\delta)}
$$

for all $n \geq n_{0}(\delta)$, which is the good upper bound we need. In particular, using $\bar{W}_{s}^{t}=\bar{W}_{t+s}-\bar{W}_{t}$ for the time-shifted path, this shows that for all $n \geq n_{0}(\delta)$,

$$
\begin{aligned}
& \mathbf{P}\left(\hat{z}_{1}\left(W_{t}, \varepsilon_{n}\right) \leq(1-\delta)^{2}\right) \\
& \quad=\mathbf{P}\left(\mu_{[-t, 1-t]}^{\bar{W}^{t}}\left(B\left(0, \varepsilon_{n}\right)\right) \leq(1-\delta)^{2} \varepsilon_{n}^{2} /\left|\log \varepsilon_{n}\right|\right) \leq \varepsilon_{n}^{2 /(1-\delta)}
\end{aligned}
$$

provided that

$$
(1-\delta)^{2} \varepsilon_{n}^{2} /\left|\log \varepsilon_{n}\right| \leq t \leq 1-(1-\delta)^{2} \varepsilon_{n}^{2} /\left|\log \varepsilon_{n}\right| .
$$

On the other hand, if $0 \leq t \leq 1$ but condition (6.9) does not hold, (i.e., for $t$ close to 0 or 1), we can no longer use the good upper bound (6.8), but must work with the following bound which comes from (6.5):

$$
\mathbf{P}\left(\hat{z}_{1}\left(W_{t}, \varepsilon_{n}\right) \leq(1-\delta)^{2}\right) \leq \mathbf{P}\left(\tau_{d}(1) \leq(1-\delta)^{2} /\left|\log \varepsilon_{n}\right|\right) \leq \varepsilon_{n}^{0.5 /(1-\delta)^{2}}
$$

for all $n \geq n_{1}(\delta)$, some $n_{1}(\delta)<\infty$.

To apply these estimates for proving (6.2), take $k=k(\delta)=20(1-\delta)^{2} / \delta^{2}$ to be an integer, $\rho_{n}=(1-\delta)^{2} \varepsilon_{n}^{2} /\left(\left|\log \varepsilon_{n}\right| k(\delta)\right)=\delta^{2} \varepsilon_{n-1}^{2} /\left(20\left|\log \varepsilon_{n}\right|\right), N_{n}=\left\lfloor\rho_{n}^{-1}\right\rfloor$ and $t_{i, n}=i \rho_{n}, i=1, \ldots, N_{n}$. On the one hand, by Lévy's uniform modulus of continuity, we have that a.s. for some finite $n_{0}=n_{0}(\omega) \geq \delta^{-1}$ and all $n \geq n_{0}$,

$$
\max _{i=1}^{N_{n}} \sup _{|s|<\rho_{n}}\left|W_{t_{i, n}+s}-W_{t_{i, n}}\right|<\delta \varepsilon_{n-1}
$$


which implies that

$$
\inf _{t \in[0,1]} \hat{z}_{1}\left(W_{t}, \varepsilon_{n-1}\right) \geq(1-\delta)^{3} \min _{i=1}^{N_{n}} \hat{z}_{1}\left(W_{t_{i, n}}, \varepsilon_{n}\right) .
$$

On the other hand, we see that condition (6.9) is satisfied by all but the first and last $k$ points of the form $t=t_{i, n}, i=1, \ldots, N_{n}$. Hence, using the good upper bound (6.8) for those $t_{i, n}$, and the bound (6.10) for the remaining $2 k$ $t_{i, n}$ 's we have

$$
\begin{aligned}
\mathbf{P}\left(\min _{i=1}^{N_{n}} \hat{z}_{1}\left(W_{t_{i, n}}, \varepsilon_{n}\right) \leq(1-\delta)^{2}\right) & \leq \sum_{i=1}^{N_{n}} \mathbf{P}\left(\hat{z}_{1}\left(W_{t_{i, n}}, \varepsilon_{n}\right) \leq(1-\delta)^{2}\right) \\
& \leq 2 k \varepsilon_{n}^{0.5 /(1-\delta)^{2}}+N_{n} \varepsilon_{n}^{2 /(1-\delta)} \leq \varepsilon_{n}^{2 \delta}
\end{aligned}
$$

Since $\varepsilon_{n}^{2 \delta}$ is summable, combining (6.11) and (6.12) yields (6.2) by an application of the Borel-Cantelli lemma.

Turning to prove (6.3), let now $\gamma_{n}=(1+\delta)^{5} \varepsilon_{n}^{2} /\left(2\left|\log \varepsilon_{n}\right|\right), \rho_{n}=\varepsilon_{n}^{2-5.6 \delta}$ and $n$ large enough for $\rho_{n} \geq \gamma_{n}$. (Our choice of the constant 5.6 will become clear at the end of the proof). Let $\varepsilon_{n}^{\prime}=(1+\delta) \varepsilon_{n}$ and consider the event $\mathscr{A}=\mathscr{A}^{+} \cap \mathscr{A}^{-}$, where

$$
\mathscr{A}^{+}=\left\{\tau_{d}\left(\varepsilon_{n}^{\prime}\right) \leq \gamma_{n}, \inf _{s \in\left[0, \rho_{n}\right]}\left|W_{\tau_{d}\left(\varepsilon_{n}^{\prime}\right)+s}\right| \geq \varepsilon_{n},\left|W_{\tau_{d}\left(\varepsilon_{n}^{\prime}\right)+\rho_{n}}\right| \geq \varepsilon_{n}^{1-\delta}\right\}
$$

and

$$
\mathscr{A}^{-}=\left\{\bar{\tau}_{d}\left(\varepsilon_{n}^{\prime}\right) \leq \gamma_{n}, \inf _{s \in\left[0, \rho_{n}\right]}\left|\bar{W}_{-\bar{\tau}_{d}\left(\varepsilon_{n}^{\prime}\right)-s}\right| \geq \varepsilon_{n},\left|\bar{W}_{-\bar{\tau}_{d}\left(\varepsilon_{n}^{\prime}\right)-\rho_{n}}\right| \geq \varepsilon_{n}^{1-\delta}\right\} .
$$

By the strong Markov property and symmetry,

$$
\begin{aligned}
\mathbf{P}\left(\mathscr{A}^{+}\right) & =\mathbf{P}\left(\mathbf{P}^{W_{\tau_{d}\left(\varepsilon_{n}^{\prime}\right)}}\left(\left|W_{\rho_{n}}\right| \geq \varepsilon_{n}^{1-\delta}, \inf _{s \in\left[0, \rho_{n}\right]}\left|W_{s}\right| \geq \varepsilon_{n}\right) ; \tau_{d}\left(\varepsilon_{n}^{\prime}\right) \leq \gamma_{n}\right) \\
& =\mathbf{P}\left(\tau_{d}\left(\varepsilon_{n}^{\prime}\right) \leq \gamma_{n}\right) \mathbf{P}^{x_{0}}\left(\left|W_{\rho_{n}}\right| \geq \varepsilon_{n}^{1-\delta}, \inf _{s \in\left[0, \rho_{n}\right]}\left|W_{s}\right| \geq \varepsilon_{n}\right),
\end{aligned}
$$

for any $x_{0}$ with $\left|x_{0}\right|=\varepsilon_{n}^{\prime}$.

By Brownian scaling, $\mathbf{P}\left(\tau_{d}\left(\varepsilon_{n}^{\prime}\right) \leq \gamma_{n}\right)=\mathbf{P}\left(\tau_{d}(1) \leq(1+\delta)^{3} /\left(2\left|\log \varepsilon_{n}\right|\right)\right.$, so that using (6.5) and $(1+\delta)^{-3}=1-3 \delta+O\left(\delta^{2}\right)$ we get

$$
c_{3} \varepsilon_{n}^{1-2.9 \delta} \leq \mathbf{P}\left(\tau_{d}\left(\varepsilon_{n}^{\prime}\right) \leq \gamma_{n}\right) \leq c_{4} \varepsilon_{n}^{1-3.1 \delta}
$$

for some $c_{3}, c_{4}>0, \delta$ small and all $n$ large enough. Since

$$
\mathbf{P}^{x}\left(\inf _{s \geq 0}\left|W_{s}\right|<\varepsilon\right)=\left(\frac{\varepsilon}{|x|}\right)^{d-2},
$$

whenever $|x|>\varepsilon$, we have, with $\left|x_{0}\right|=\varepsilon_{n}^{\prime}=(1+\delta) \varepsilon_{n}$,

$$
\mathbf{P}^{x_{0}}\left(\inf _{s \geq 0}\left|W_{s}\right| \geq \varepsilon_{n}\right)=1-(1+\delta)^{-(d-2)},
$$


hence

$$
1-(1+\delta)^{-(d-2)} \leq \mathbf{P}^{x_{0}}\left(\inf _{s \in\left[0, \rho_{n}\right]}\left|W_{s}\right| \geq \varepsilon_{n}\right) \leq 1
$$

while

$$
\begin{aligned}
\mathbf{P}^{x_{0}}\left(\left|W_{\rho_{n}}\right| \leq \varepsilon_{n}^{1-\delta}\right) & =\mathbf{P}\left(\left|x_{0}+\varepsilon_{n}^{1-2.8 \delta} W_{1}\right| \leq \varepsilon_{n}^{1-\delta}\right) \\
& =\mathbf{P}\left(\left|\varepsilon_{n}^{2.8 \delta}\left(x_{0} / \varepsilon_{n}\right)+W_{1}\right| \leq \varepsilon_{n}^{1.8 \delta}\right) \rightarrow 0
\end{aligned}
$$

since $\left|x_{0} / \varepsilon_{n}\right|=1+\delta$, independent of $n$. Putting this all together and noting that $\mathbf{P}(\mathscr{A})=\mathbf{P}\left(\mathscr{A}^{+}\right) \mathbf{P}\left(\mathscr{L}^{-}\right)=\mathbf{P}\left(\mathscr{A}^{+}\right)^{2}$ shows that

$$
c \varepsilon_{n}^{2-5.8 \delta} \leq \mathbf{P}(\mathscr{A}) \leq c^{\prime} \varepsilon_{n}^{2-6.2 \delta}
$$

for $c, c^{\prime}>0$ independent of $n$.

With $t_{i, n}=4 i \rho_{n}$ and $N_{n}=\left[\left(4 \rho_{n}\right)^{-1}\right]=\left[0.25 \varepsilon_{n}^{-2+5.6 \delta}\right]$, set $\mathscr{A}_{i}=\mathscr{A} \circ \theta_{t_{i, n}}$, that is, the event $\mathscr{A}$ for the shifted path $W^{t_{i, n}}\left(\bar{W}^{t_{i, n}}\right)$. By the strong Markov property, for any $i=1, \ldots, N_{n}$,

$$
\mathbf{P}\left(\hat{z}_{\infty}\left(W_{t_{i, n}}, \varepsilon_{n}\right) \geq(1+\delta)^{5} \mid \mathscr{A}_{i}\right) \leq 2 \max _{\left|x_{0}\right| \geq \varepsilon_{n}^{1-\delta}} \mathbf{P}^{x_{0}}\left(\inf _{t \geq 0}\left|W_{t}\right|<\varepsilon_{n}\right) \leq 2 \varepsilon_{n}^{(d-2) \delta},
$$

where (6.14) was used in the second inequality. Hence, by the independence of the events $\left\{\mathscr{L}_{i}\right\}_{i=1}^{N_{n}}$,

$$
\begin{aligned}
& \mathbf{P}\left(\min _{i=1}^{N_{n}} \hat{z}_{\infty}\left(W_{t_{i, n}}, \varepsilon_{n}\right) \geq(1+\delta)^{5}\right) \\
& \quad \leq(1-\mathbf{P}(\mathscr{A}))^{N_{n}}+\sum_{i=1}^{N_{n}} \mathbf{P}\left(\hat{z}_{\infty}\left(W_{t_{i, n}}, \varepsilon_{n}\right) \geq(1+\delta)^{5}, \mathscr{A}_{i}\right) \\
& \quad \leq \exp \left(-\mathbf{P}(\mathscr{A}) N_{n}\right)+\sum_{i=1}^{N_{n}} \mathbf{P}\left(\hat{z}_{\infty}\left(W_{t_{i, n}}, \varepsilon_{n}\right) \geq(1+\delta)^{5} \mid \mathscr{A}_{i}\right) \mathbf{P}\left(\mathscr{A}_{i}\right) \\
& \quad \leq \exp \left(-c \varepsilon_{n}^{-0.2 \delta}\right)+c^{\prime} \varepsilon_{n}^{-0.6 \delta} \varepsilon_{n}^{(d-2) \delta} \\
& \quad \leq \exp \left(-c \varepsilon_{n}^{-0.2 \delta}\right)+c^{\prime} \varepsilon_{n}^{0.4 \delta}
\end{aligned}
$$

and (6.3) follows by an application of the Borel-Cantelli lemma. (One can see now the reason for choosing the constant 5.6 above. With more care, we could have chosen $\rho_{n}=\varepsilon_{n}^{2-q \delta}$ with $5<q<6$.) This completes the proof of Theorem 1.3 .

\section{Almost sure convergence of exponential moments.}

Proof of TheOREM 1.4. For any Borel function $f:[a, b] \rightarrow \mathbb{R}^{d}$, we use $\mu_{a, b}^{f}$ to denote its occupation measure,

$$
\mu_{a, b}^{f}(A)=\int_{a}^{b} \mathbf{1}_{A}\left(f_{t}\right) d t
$$


for all Borel sets $A \subseteq \mathbb{R}^{d}$. We use the abbreviations $\mu_{T}^{f}=\mu_{0, T}^{f}$ and $\bar{\mu}_{T}^{f}=\mu_{-T, T}^{f}$.

As a first step in proving Theorem 1.4, we rewrite things so that we deal only with occupation measures of $B(0,1)$. Writing $W_{t}^{\varepsilon}=\varepsilon^{-1} W_{\varepsilon^{2} t}$ and $W_{s}^{\varepsilon, t}=$ $W_{t+s}^{\varepsilon}-W_{t}^{\varepsilon}$ with similar notation for $\bar{W}$ we have

$$
\begin{aligned}
\mu_{1}^{W}\left(B\left(W_{\varepsilon^{2} t}, \varepsilon\right)\right) & =\int_{0}^{1} \mathbf{1}_{\left\{\left|W_{s}-W_{\varepsilon^{2} t}\right| \leq \varepsilon\right\}} d s=\varepsilon^{2} \int_{0}^{1 / \varepsilon^{2}} \mathbf{1}_{\left\{\left|W_{\varepsilon^{2} s}-W_{\varepsilon^{2} t}\right| \leq \varepsilon\right\}} d s \\
& =\varepsilon^{2} \int_{0}^{1 / \varepsilon^{2}} \mathbf{1}_{\left\{\left|W_{s}^{\varepsilon}-W_{t}^{\varepsilon}\right| \leq 1\right\}} d s=\varepsilon^{2} \int_{0}^{1 / \varepsilon^{2}} \mathbf{1}_{B(0,1)}\left(W_{s}^{\varepsilon}-W_{t}^{\varepsilon}\right) d s
\end{aligned}
$$

and consequently,

$$
\begin{aligned}
\int_{0}^{1} \exp \left(\theta \mu_{1}^{W}\left(B\left(W_{t}, \varepsilon\right)\right) / \varepsilon^{2}\right) d t \\
=\varepsilon^{2} \int_{0}^{1 / \varepsilon^{2}} \exp \left(\theta \mu_{1}^{W}\left(B\left(W_{\varepsilon^{2} t}, \varepsilon\right)\right) / \varepsilon^{2}\right) d t \\
=\varepsilon^{2} \int_{0}^{1 / \varepsilon^{2}} \exp \left(\theta \int_{0}^{1 / \varepsilon^{2}} \mathbf{1}_{B(0,1)}\left(W_{s}^{\varepsilon}-W_{t}^{\varepsilon}\right) d s\right) d t \\
\leq \varepsilon^{2} \int_{0}^{1 / \varepsilon^{2}} \exp \left(\theta \int_{-\infty}^{\infty} \mathbf{1}_{B(0,1)}\left(\bar{W}_{s}^{\varepsilon}-\bar{W}_{t}^{\varepsilon}\right) d s\right) d t \\
=\varepsilon^{2} \int_{0}^{1 / \varepsilon^{2}} \exp \left(\theta \int_{-\infty}^{\infty} \mathbf{1}_{B(0,1)}\left(\bar{W}_{s}^{\varepsilon, t}\right) d s\right) d t \\
=\varepsilon^{2} \int_{0}^{1 / \varepsilon^{2}} \exp \left(\theta \bar{\mu}_{\infty}^{\varepsilon, t}(B(0,1))\right) d t .
\end{aligned}
$$

Hence for each $\theta<q_{d}^{2} / 2$ and any subsequence $\varepsilon_{m} \rightarrow 0$, in order to show that

$$
\begin{gathered}
\limsup _{m \rightarrow \infty} \int_{0}^{1} \exp \left(\theta \mu_{1}^{W}\left(B\left(W_{t}, \varepsilon_{m}\right)\right) / \varepsilon_{m}^{2}\right) d t \\
\leq \mathbb{E}\left(\exp \left(\theta \bar{\mu}_{\infty}^{\bar{W}}(B(0,1))\right)\right) \quad \text { a.s., }
\end{gathered}
$$

it suffices to show that

$$
\begin{gathered}
\lim _{m \rightarrow \infty} \varepsilon_{m}^{2} \int_{0}^{1 / \varepsilon_{m}^{2}} \exp \left(\theta \bar{\mu}_{\infty}^{\bar{W}^{\varepsilon_{m}, t}}(B(0,1))\right) d t \\
=\mathbb{E}\left(\exp \left(\theta \bar{\mu}_{\infty}^{\bar{W}}(B(0,1))\right)\right) \quad \text { a.s. }
\end{gathered}
$$

For any $1<p<2$ such that $p \theta<q_{d}^{2} / 2$, (7.3) will follow with $\varepsilon_{m}=m^{-2 /(p-1)}$ from the Borel-Cantelli lemma, Chebyshev's inequality and the following lemma. For notational convienience we shall sometimes write $\bar{W}\left(n^{-1}, t\right)$ for $\bar{W}^{n^{-1}, t}$. 
LEMMA 7.1. For $\theta<q_{d}^{2} / 2$, there exists $c=c_{d, \theta}$ finite, such that for all $n$,

$$
\begin{aligned}
& \| \frac{1}{n^{2}} \int_{0}^{n^{2}} \exp \left(\theta \bar{\mu}_{\infty}^{\bar{W}\left(n^{-1}, t\right)}(B(0,1))\right) d t \\
& -\frac{1}{n^{2}} \int_{0}^{n^{2}} \exp \left(\theta \bar{\mu}_{n}^{\bar{W}\left(n^{-1}, t\right)}(B(0,1))\right) d t \|_{1} \leq c n^{-(d / 2-1)},
\end{aligned}
$$

and for any $1<p<2$ such that $p \theta<q_{d}^{2} / 2$, there exists $c=c_{p, d, \theta}$ finite, such that for all $n$,

$$
\begin{aligned}
\| \frac{1}{n^{2}} \int_{0}^{n^{2}} \exp \left(\theta \bar{\mu}_{n}^{\bar{W}\left(n^{-1}, t\right)}(B(0,1))\right) d t \\
\quad-\mathbb{E}\left(\exp \left(\theta \bar{\mu}_{\infty}^{\bar{W}}(B(0,1))\right)\right) \|_{p} \leq c n^{-(1-1 / p) .}
\end{aligned}
$$

Before proving this lemma we first use it to show that for any $1<p<2$ such that $p \theta<q_{d}^{2} / 2$, and with $\varepsilon_{m}=m^{-2 /(p-1)}$,

$$
\begin{gathered}
\liminf _{m \rightarrow \infty} \int_{0}^{1} \exp \left(\theta \mu_{1}^{W}\left(B\left(W_{t}, \varepsilon_{m}\right)\right) / \varepsilon_{m}^{2}\right) d t \\
\geq \mathbb{E}\left(\exp \left(\theta \bar{\mu}_{\infty}^{\bar{W}}(B(0,1))\right)\right) \quad \text { a.s. }
\end{gathered}
$$

Note that for any $n \leq t \leq n^{2}-n$,

$$
\begin{aligned}
\int_{0}^{n^{2}} \mathbf{1}_{B(0,1)}\left(W_{s}^{n^{-1}}-W_{t}^{n^{-1}}\right) d s & =\int_{-t}^{n^{2}-t} \mathbf{1}_{B(0,1)}\left(\bar{W}_{s}^{n^{-1}, t}\right) d s \\
& \geq \int_{-n}^{n} \mathbf{1}_{B(0,1)}\left(\bar{W}_{s}^{n^{-1}, t}\right) d s \\
& =\bar{\mu}_{n}^{\bar{W}\left(n^{-1}, t\right)}(B(0,1)) .
\end{aligned}
$$

Hence from (7.1),

$$
\begin{aligned}
\int_{0}^{1} \exp \left(\theta \mu_{1}^{W}\left(B\left(W_{t}, n^{-1}\right)\right) / n^{-2}\right) d t \\
\quad=\frac{1}{n^{2}} \int_{0}^{n^{2}} \exp \left(\theta \int_{0}^{n^{2}} \mathbf{1}_{B(0,1)}\left(W_{s}^{n^{-1}}-W_{t}^{n^{-1}}\right) d s\right) d t \\
\quad \geq \frac{1}{n^{2}} \int_{n}^{n^{2}-n} \exp \left(\theta \bar{\mu}_{n}^{\bar{W}\left(n^{-1}, t\right)}(B(0,1))\right) d t
\end{aligned}
$$

Equation (7.6) then follows by using Lemma 7.1 as before and noting that

$$
\begin{aligned}
& \| \frac{1}{n^{2}} \int_{0}^{n^{2}} \exp \left(\theta \bar{\mu}_{n}^{\bar{W}\left(n^{-1}, t\right)}(B(0,1))\right) d t \\
& -\frac{1}{n^{2}} \int_{n}^{n^{2}-n} \exp \left(\theta \bar{\mu}_{n}^{\bar{W}\left(n^{-1}, t\right)}(B(0,1))\right) d t \|_{1}
\end{aligned}
$$




$$
\begin{aligned}
\leq & \left\|\frac{1}{n^{2}} \int_{0}^{n} \exp \left(\theta \bar{\mu}_{n}^{\bar{W}\left(n^{-1}, t\right)}(B(0,1))\right) d t\right\|_{1} \\
& +\left\|\frac{1}{n^{2}} \int_{n^{2}-n}^{n^{2}} \exp \left(\theta \bar{\mu}_{n}^{\bar{W}\left(n^{-1}, t\right)}(B(0,1))\right) d t\right\|_{1} \\
\leq & \frac{1}{n^{2}} \int_{0}^{n}\left\|\exp \left(\theta \bar{\mu}_{n}^{\bar{W}\left(n^{-1}, t\right)}(B(0,1))\right)\right\|_{1} d t \\
& +\frac{1}{n^{2}} \int_{n^{2}-n}^{n^{2}}\left\|\exp \left(\theta \bar{\mu}_{n}^{\bar{W}\left(n^{-1}, t\right)}(B(0,1))\right)\right\|_{1} d t \\
\leq & 2 n^{-1}\left\|\exp \left(\theta \bar{\mu}_{\infty}^{\bar{W}}(B(0,1))\right)\right\|_{1} .
\end{aligned}
$$

Since $\mu_{1}^{W}\left(B\left(W_{t}, \varepsilon\right)\right)$ is monotone in $\varepsilon$ and $\lim _{m \rightarrow \infty} \varepsilon_{m+1} / \varepsilon_{m}=1$, the proof of Theorem 1.4 now follows from (7.2), (7.6) and a simple interpolation argument.

Proof of Lemma 7.1. Equation (7.4) will follow from

$$
\begin{gathered}
\left\|\frac{1}{n^{2}} \int_{0}^{n^{2}} \exp \left(\theta \bar{\mu}_{\infty}^{\bar{W}\left(n^{-1}, t\right)}(B(0,1))\right) d t-\frac{1}{n^{2}} \int_{0}^{n^{2}} \exp \left(\theta \bar{\mu}_{n}^{\bar{W}\left(n^{-1}, t\right)}(B(0,1))\right) d t\right\|_{1} \\
\leq \frac{1}{n^{2}} \int_{0}^{n^{2}}\left\|\exp \left(\theta \bar{\mu}_{\infty}^{\bar{W}\left(n^{-1}, t\right)}(B(0,1))\right)-\exp \left(\theta \bar{\mu}_{n}^{\bar{W}\left(n^{-1}, t\right)}(B(0,1))\right)\right\|_{1} d t
\end{gathered}
$$

and the following lemma.

LEMMA 7.2. For any $\theta<q_{d}^{2} / 2$, there exists $c=c_{d, \theta}$ finite such that for any $\varepsilon>0$,

$$
\left\|\exp \left(\theta \bar{\mu}_{\infty}^{\bar{W}}(B(0,1))\right)-\exp \left(\theta \bar{\mu}_{1 / \varepsilon}^{\bar{W}}(B(0,1))\right)\right\|_{1} \leq c \varepsilon^{d / 2-1}
$$

As for (7.5), we first rewrite

$$
\begin{aligned}
& \frac{1}{n^{2}} \int_{0}^{n^{2}} \exp \left(\theta \bar{\mu}_{n}^{\bar{W}\left(n^{-1}, t\right)}(B(0,1))\right) d t \\
& \quad=\frac{1}{n} \sum_{k=0}^{n-1} \frac{1}{n} \int_{k n}^{(k+1) n} \exp \left(\theta \bar{\mu}_{n}^{\bar{W}\left(n^{-1}, t\right)}(B(0,1))\right) d t \\
& \quad=\frac{1}{n} \sum_{k=0}^{n-1} I_{n, k},
\end{aligned}
$$

where

$$
I_{n, k}=\frac{1}{n} \int_{k n}^{(k+1) n} \exp \left(\theta \bar{\mu}_{n}^{\bar{W}\left(n^{-1}, t\right)}(B(0,1))\right) d t .
$$

Unraveling the definitions, we see that for each fixed $n$, the $I_{n, k} ; 0 \leq k \leq$ $n$ are identically distributed, and $I_{n, k}$ is measurable with respect to the $\sigma$ - 
algebra generated by $\left\{\bar{W}_{t+s}^{n^{-1}}-\bar{W}_{t}^{n^{-1}} ; k n \leq t \leq(k+1) n ;-n \leq s \leq n\right\}$. Hence $I_{n, k}, I_{n, k^{\prime}}$ are independent as soon as $\left|k-k^{\prime}\right| \geq 3$. Thus we can write

$$
\frac{1}{n} \sum_{k=0}^{n} I_{n, k}=\frac{1}{n} \sum_{k=0}^{n / 3} I_{n, 3 k}+\frac{1}{n} \sum_{k=0}^{n / 3} I_{n, 1+3 k}+\frac{1}{n} \sum_{k=0}^{n / 3} I_{n, 2+3 k},
$$

where each of the three sums on the right-hand side is now a sum of i.i.d. random variables. Furthermore,

$$
\begin{aligned}
\mathbb{E}\left(I_{n, k}\right) & =\frac{1}{n} \int_{k n}^{(k+1) n} \mathbb{E}\left(\exp \left(\theta \bar{\mu}_{n}^{\bar{W}\left(n^{-1}, t\right)}(B(0,1))\right)\right) d t \\
& =\mathbb{E}\left(\exp \left(\theta \bar{\mu}_{n}^{\bar{W}}(B(0,1))\right)\right) .
\end{aligned}
$$

Using Lemma 7.2, to complete the proof of Lemma 7.1, it now suffices to note that for any $1<p<2$ such that $p \theta<q_{d}^{2} / 2$ we have the following bounds, where the first inequality comes from the Marcinkiewicz-Zygmund inequality (see, e.g., [25], page 341), where our condition $p \theta<q_{d}^{2} / 2$ guarantees that $\left.I_{n, k} \in L^{p}\right)$, and the second inequality comes from the fact that $|a+b|^{p / 2} \leq$ $|a|^{p / 2}+|b|^{p / 2}$ (since $p<2$ ),

$$
\begin{aligned}
& \mathbb{E}\left(\left|\frac{1}{n / 3} \sum_{k=0}^{n / 3}\left(I_{n, i+3 k}-\mathbb{E}\left(I_{n, i+3 k}\right)\right)\right|^{p}\right) \\
& \leq \frac{c}{n^{p}} \mathbb{E}\left(\left|\sum_{k=0}^{n / 3}\left(I_{n, i+3 k}-\mathbb{E}\left(I_{n, i+3 k}\right)\right)^{2}\right|^{p / 2}\right) \leq c n^{-(p-1)}
\end{aligned}
$$

for $i=0,1,2$.

PRoOF OF LEMMA 7.2. Let $p_{r}(x)=(2 \pi r)^{-d / 2} \exp \left(-|x|^{2} / 2 r\right)$ and $u^{0}(x)=$ $\int_{0}^{\infty} p_{r}(x) d r=c_{d} /|x|^{d-2}$ denote the zero-potential of $W_{t}$. Let $\Lambda_{d}$ denote the norm of

$$
K f(x)=\int_{B(0,1)} u^{0}(x-y) f(y) d y
$$

considered as an operator from $L^{2}(B(0,1), d x)$ to itself, recalling from [3] that $\Lambda_{d}^{-1}=q_{d}^{2} / 2$ is the first eigenvalue of $(1 / 2) \Delta$ in the unit ball of $\mathbb{R}^{d-2}$ (not $\left.\mathbb{R}^{d}\right)$ with Dirichlet boundary conditions. We claim that $K^{i} u^{0} \in L^{2}(B(0,1), d x)$ for sufficiently large $i$ ( $i=[d / 2]$ will do). To see this, note that $u^{0} \in L^{2}\left(B(0,1), d^{3} x\right)$ for $d=3$, while for $d=4$, by scaling, $K u^{0}(x) \leq c \int \mid x-$ $\left.y\right|^{-2}|y|^{-2} d^{4} y \leq c \log (1 /|x|) \in L^{2}\left(B(0,1), d^{4} x\right)$. When $d>4$ we first note, again by scaling, that $\int|x-y|^{-(d-2)}|y|^{-k} d^{d} y \leq c|x|^{-(k-2)}$ for any $k>2$, and we can repeat this argument until we find that $K^{j} u^{0}(x) \leq c|y|^{-k}$ with $1 \leq k \leq 2$ for some $j$. Noting that for such $k$ we have $|y|^{-k} \in L^{2}\left(\bar{B}(0,1), d^{d} x\right)$ completes the verification of our claim. It follows from this in particular, for some $\kappa_{d}<\infty$ and all $i$,

$$
\int_{B(0,1)} K^{i} u^{0}(x) d x \leq \kappa_{d}\left(\Lambda_{d}\right)^{i}
$$


Setting $t_{0}=0, x_{0}=0$, we first bound the following moments for $m=$ $1,2, \ldots$ :

$$
\begin{aligned}
\frac{1}{m !} & \mathbb{E} \\
\quad & =\frac{1}{m} \sum_{i=1}^{\infty} \int_{B(0,1)^{m}} \int_{\substack{0 \leq t_{1} \leq \cdots \leq t_{m}<\infty \\
\varepsilon^{-1} \leq t_{i}}} \prod_{j=1}^{m} p_{t_{j}-t_{j-1}}\left(x_{j}-x_{j-1}\right) d t_{1} \cdots d t_{m} d x_{1} \cdots d x_{m} \\
& =\frac{1}{m} \sum_{i=1}^{m} \int_{B(0,1)^{m}} \int_{\varepsilon^{-1} \leq \sum_{j=1}^{i} r_{j}} \prod_{j=1}^{m-1} p_{r_{j}}\left(x_{j}-x_{j-1}\right) d r_{1} \cdots d r_{m} d x_{1} \cdots d x_{m} \\
& \leq \sum_{i=1}^{m} \int_{B(0,1)^{m}} \int_{(m \varepsilon)^{-1} \leq r_{i}} \prod_{j=1}^{m} p_{r_{j}}\left(x_{j}-x_{j-1}\right) d r_{1} \cdots d r_{m} d x_{1} \cdots d x_{m} \\
& =\sum_{i=1}^{m} \int_{B(0,1)^{m}} \prod_{\substack{j=1 \\
j \neq i}}^{m} u^{0}\left(x_{j}-x_{j-1}\right) \int_{(m \varepsilon)^{-1}}^{\infty} p_{r_{i}}\left(x_{i}-x_{i-1}\right) d r_{i} d x_{1} \cdots d x_{m} \\
& \leq\left(\int_{(m \varepsilon)^{-1}}^{\infty} p_{r}(0) d r\right) \sum_{i=1}^{m} \int_{B(0,1)^{m}} \prod_{\substack{j=1 \\
j \neq i}}^{m} u^{0}\left(x_{j}-x_{j-1}\right) d x_{1} \cdots d x_{m} \\
& =k_{d}(m \varepsilon)^{d / 2-1}\left[\left(1, K^{m-1} 1\right)_{B(0,1)}\right. \\
& \left.\leq c_{d} \varepsilon^{d / 2-1} m^{d / 2} \Lambda_{d}^{m-1}, \sum_{i=2}^{m}\left(\int_{B(0,1)} K^{i-2} u^{0}\left(x_{i-1}\right) d x_{i-1}\right)\left(1, K^{m-i} 1\right)_{B(0,1)}\right]
\end{aligned}
$$

where $(\cdot, \cdot)_{B(0,1)}$ denotes the inner product in $L^{2}(B(0,1), d x)$ and (7.7) was used in the last inequality. With $c_{d}$ independent of

$m$ and $g_{d, \theta}=\mathbb{E}\left(\exp \left(\theta \mu_{\infty}^{W}(B(0,1))\right)\right)$ finite, it follows that

$$
\begin{aligned}
\| \exp & \left(\theta \bar{\mu}_{\infty}^{\bar{W}}(B(0,1))\right)-\exp \left(\theta \bar{\mu}_{1 / \varepsilon}^{\bar{W}}(B(0,1))\right) \|_{1} \\
= & \mathbb{E}\left(\exp \left(\theta \bar{\mu}_{\infty}^{\bar{W}}(B(0,1))\right)\right)-\mathbb{E}\left(\exp \left(\theta \bar{\mu}_{1 / \varepsilon}^{\bar{W}}(B(0,1))\right)\right) \\
= & \left\{\mathbb{E}\left(\exp \left(\theta \mu_{\infty}^{W}(B(0,1))\right)\right)\right\}^{2}-\left\{\mathbb{E}\left(\exp \left(\theta \mu_{1 / \varepsilon}^{W}(B(0,1))\right)\right)\right\}^{2} \\
& \leq 2 g_{d, \theta}\left|\mathbb{E}\left(\exp \left(\theta \mu_{\infty}^{W}(B(0,1))\right)\right)-\mathbb{E}\left(\exp \left(\theta \mu_{1 / \varepsilon}^{W}(B(0,1))\right)\right)\right| \\
\leq & 2 g_{d, \theta} \theta \mathbb{E}\left(\left|\mu_{\infty}^{W}(B(0,1))-\mu_{1 / \varepsilon}^{W}(B(0,1))\right| \exp \left(\theta \mu_{\infty}^{W}(B(0,1))\right)\right) \\
& =2 g_{d, \theta} \sum_{m=0}^{\infty} \frac{\theta^{m+1}}{m !} \mathbb{E}\left(\int_{1 / \varepsilon}^{\infty} \mathbf{1}_{B(0,1)}\left(W_{r}\right) d r\left(\int_{0}^{\infty} \mathbf{1}_{B(0,1)}\left(W_{s}\right) d s\right)^{m}\right) \\
& \leq 2 \theta g_{d, \theta} c_{d} \varepsilon^{d / 2-1} \sum_{m=0}^{\infty}(m+1)^{d / 2+1}\left(\theta \Lambda_{d}\right)^{m} \leq c_{d, \theta} \varepsilon^{d / 2-1}
\end{aligned}
$$


for any $\theta<\Lambda_{d}^{-1}$, as needed to complete the proof of Lemma 7.2 and hence of Theorem 1.4.

\section{The coarse multifractal spectrum.}

Proof of Corollary 1.5. The lower bound in Corollary 1.5 is an immediate consequence of (1.10) and Chebyshev's inequality. Turning to the corresponding upper bound, fix $a \in\left(0,2 / \theta^{*}\right)$. Choosing $\delta \in(0,1 / 4)$ such that $\eta=2-a \theta^{*}(1+3 \delta)>0$ and $\varepsilon_{n}$ as in (5.2), leads [see (6.1)] to

$$
\begin{aligned}
& \limsup _{\varepsilon \rightarrow 0} \frac{\log \mathscr{L} e b\left\{0 \leq t \leq 1 \mid z_{1}\left(W_{t}, \varepsilon\right) \geq a\right\}}{\log \varepsilon} \\
& \quad \leq \limsup _{n \rightarrow \infty} \frac{\log \mathscr{L} e b\left\{0 \leq t \leq 1 \mid z_{1}\left(W_{t}, \varepsilon_{n}\right) \geq a / 1-\delta\right\}}{\log \varepsilon_{n-1}} .
\end{aligned}
$$

Let $W_{s}^{t}=W_{t+s}-W_{t}, \delta_{n}=\varepsilon_{n}^{2}\left|\log \varepsilon_{n}\right|^{6}$ and $\beta_{n}=1-2\left|\log \varepsilon_{n}\right|^{-2}$. The random variables $Y_{i}^{(n)}=\mu_{\delta_{n}}^{W^{i \delta_{n}}}\left(B\left(0, \beta_{n} \varepsilon_{n}\right)\right) / h\left(\varepsilon_{n}\right), i=1, \ldots, \delta_{n}^{-1}-1$ are i.i.d. The Localization Lemma implies that for some $c>0$ and all $n$ large enough,

$$
p_{n}^{*}:=\mathbf{P}\left(Y^{(n)} \geq a /(1-\delta)\right) \geq c \varepsilon_{n}^{a \theta^{*}(1+2 \delta)} .
$$

Thus, by standard tail estimates for the Binomial $\left(\delta_{n}^{-1}-1, p_{n}^{*}\right)$, for all $n$ large enough,

$$
\mathbf{P}\left(\left|\left\{i: Y_{i}^{(n)} \geq a /(1-\delta)\right\}\right| \leq \varepsilon_{n}^{-\eta}\right) \leq \exp \left(-\varepsilon_{n}^{-\eta}\right),
$$

since $(1-\delta)^{-1} \leq 1+2 \delta$. It follows that a.s., for all $n \geq n_{0}(\omega, \delta, a)$,

$$
\left|\left\{i: Y_{i}^{(n)} \geq a /(1-\delta)\right\}\right| \geq \varepsilon_{n}^{-\eta} .
$$

Taking $\rho_{n}=\varepsilon_{n}^{2} /\left|\log \varepsilon_{n}\right|^{6}$, by Lévy's uniform modulus of continuity, we have that a.s. for some $n_{1}=n_{1}(\omega)<\infty$ and all $n \geq n_{1}$,

$$
\max _{i=1}^{\delta_{n}^{-1}-1} \sup _{|s|<\rho_{n}}\left|W_{i \delta_{n}+s}-W_{i \delta_{n}}\right|<\left(1-\beta_{n}\right) \varepsilon_{n}
$$

which together with (8.2) implies that a.s. for any $n \geq n_{2}(\omega, \delta, a)$,

$$
\mathscr{L} e b\left\{0 \leq t \leq 1 \mid z_{1}\left(W_{t}, \varepsilon_{n}\right) \geq a /(1-\delta)\right\} \geq \rho_{n}\left|\left\{i: Y_{i}^{(n)} \geq a /(1-\delta)\right\}\right| \geq \varepsilon_{n}^{2-\eta+\delta} .
$$

In view of (8.1), we have a.s.,

$$
\limsup _{\varepsilon \rightarrow 0} \frac{\log \mathscr{L} e b\left\{0 \leq t \leq 1 \mid z_{1}\left(W_{t}, \varepsilon\right) \geq a\right\}}{\log \varepsilon} \leq(1-2 \delta)^{-1 / 2}(2-\eta-\delta) .
$$

To complete the proof, consider $\delta \downarrow 0$, for which $2-\eta-\delta \rightarrow a \theta^{*}$. 


\section{Large occupation measure at all scales.}

Proof of Theorem 1.6. For $k \in(1, \infty), T<\infty$, let $\Gamma_{k}=\{x:|x| \in[1 / k, k]\}$ and

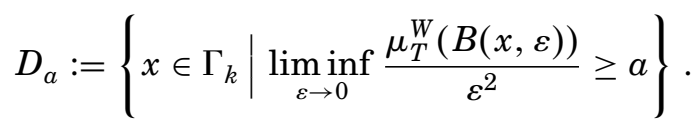

[We work with the annulus $\Gamma_{k}$ rather than the ball $B(0, k)$ because the basic bound we will use, Lemma 9.2, blows up at the origin.]

Fix $\delta>0$ and let $b=1+\delta>1$. Set $\eta_{n}=2^{-n}$ and $\delta_{n}=\eta_{n}^{1-b^{-1}}$ for $n=1,2, \ldots$, Let $\left\{x_{j}: j=1, \ldots, K_{n}\right\}, K_{n} \leq c(\delta, k, d) \eta_{n}^{-d}$, denote a maximal collection of points in $\Gamma_{k}$ such that $\inf _{l \neq j}\left|x_{l}-x_{j}\right| \geq \delta \eta_{n}$. Let $\mathscr{H}_{n}=\mathscr{H}_{n}(a, \delta, T)$ be the set of $j, 1 \leq j \leq K_{n}$, such that

$$
\inf _{\varepsilon \in\left[\eta_{n}, \delta_{n}\right]} \frac{\mu_{T}^{W}\left(B\left(x_{j}, b \varepsilon\right)\right)}{\varepsilon^{2}} \geq \frac{a}{b} .
$$

We will shortly prove that for any $\gamma>0$ we can find $\delta>0$ such that for some $c=c(a, \delta, T)<\infty$ and all $n$,

$$
\mathbb{E}\left|\mathscr{H}_{n}\right| \leq c \eta_{n}^{I_{d}(a)-2-\gamma},
$$

where $I_{d}(v)$ is defined in (1.12). Assuming this for the moment, let $\mathscr{U}_{n, j}=$ $B\left(x_{j}, \delta \eta_{n}\right)$. Then, for any $x \in \Gamma_{k}$ there exists $j \in\left\{1, \ldots, K_{n}\right\}$ such that $x \in$ $\mathscr{U}_{n, j}$ and $B(x, \varepsilon) \subseteq B\left(x_{j}, \varepsilon+\delta \eta_{n}\right) \subseteq B\left(x_{j}, b \varepsilon\right)$ for all $\varepsilon \geq \eta_{n}$. If $x \in D_{a}$ then a.s. for some $m_{1}(\omega, x, b)<\infty$ and all $n \geq m_{1}$,

$$
\inf _{\varepsilon \in\left[\eta_{n}, \delta_{n}\right]} \frac{\mu_{T}^{W}(B(x, \varepsilon))}{\varepsilon^{2}} \geq \frac{a}{b} .
$$

Therefore, $\bigcup_{n \geq m} \cup_{j \in \mathscr{H}_{n}} \mathscr{U}_{n, j}$ forms a $2 \delta \eta_{m}$-cover of $D_{a}$ for any $m \geq 1$. Since $\mathscr{U}_{n, j}$ has diameter $2 \delta \eta_{n}$, it follows from (9.2) that

$$
\begin{aligned}
\mathbb{E} \sum_{n=m}^{\infty} \sum_{j \in \mathscr{H}_{n}}\left|\mathscr{U}_{n, j}\right|^{2-I_{d}(a)+2 \gamma} \\
\quad=\sum_{n=m}^{\infty} \mathbb{E}\left|\mathscr{H}_{n}\right|\left(2 \delta \eta_{n}\right)^{2-I_{d}(a)+2 \gamma} \leq c_{2} \sum_{n=m}^{\infty} \eta_{n}^{\gamma}<\infty .
\end{aligned}
$$

Thus, $\sum_{n=m}^{\infty} \sum_{j \in \mathscr{H}_{n}}\left|\mathscr{U}_{n, j}\right|^{2-I_{d}(a)+2 \gamma}$ is finite a.s. implying that $\operatorname{dim}\left(D_{a}\right) \leq 2-$ $I_{d}(a)+2 \gamma$ a.s. for any $T<\infty, \gamma>0$. Since a.s. there exists $T_{k}=T_{k}(\omega)$ finite, such that $\left|W_{t}\right| \geq(k+1)$ for any $t \geq T_{k}$, obviously a.s. also

$$
\operatorname{dim}\left(\left\{x \in \Gamma_{k} \mid \liminf _{\varepsilon \rightarrow 0} \frac{\mu_{\infty}^{W}(B(x, \varepsilon))}{\varepsilon^{2}} \geq a\right\}\right) \leq 2-I_{d}(a)+2 \gamma .
$$

Taking $\gamma \downarrow 0$ and considering the countable union over $k=1,2 \ldots$ completes the proof of (1.14). 
To get our upper bound on packing dimension, denote by $D^{m}(a / b)$ the set of points $x \in \mathbb{R}^{d}$ such that for all $n \geq m$, we have

$$
\inf _{\varepsilon \in\left[\eta_{n}, \delta_{n}\right]} \frac{\mu_{T}^{W}(B(x, \varepsilon))}{\varepsilon^{2}} \geq \frac{a}{b} .
$$

Clearly, $\bigcup_{j \in \mathscr{H}_{n}} \mathscr{U}_{n, j}$ forms a $2 \delta \eta_{n}$-cover of $D^{m}(a / b)$ for any $n \geq m$. Thus, from (9.3),

$$
\lim _{n \rightarrow \infty} \sum_{j \in \mathscr{H}_{n}}\left|\mathscr{U}_{n, j}\right|^{2-I_{d}(a)+2 \gamma}=0 \quad \text { a.s. }
$$

Denote by $\mathscr{N}(A, \varepsilon)$ the minimal cardinality of a collection of balls of radius $\varepsilon$ that covers $A$. Recall that $\overline{\operatorname{dim}}_{\mathrm{M}}(A)$, the upper Minkowski dimension of a set $A$ (also known as the upper box-counting dimension), may be defined by

$$
\overline{\operatorname{dim}}_{\mathrm{M}}(A)=\limsup _{\varepsilon \rightarrow 0} \frac{\log \mathscr{N}(A, \varepsilon)}{|\log \varepsilon|}
$$

[see [8], (3.5)]. From (9.4) we may deduce that $\overline{\operatorname{dim}}_{\mathrm{M}}\left(D^{m}(a / b)\right) \leq 2-I_{d}(a)+2 \gamma$. Since $b>1$, necessarily

$$
D_{a} \subset \bigcup_{m \geq 1} D^{m}(a / b)
$$

and the upper bound $\operatorname{dim}_{\mathrm{p}}\left(D_{a}\right) \leq 2-I_{d}(a)+2 \gamma$ a.s. follows by [8], Proposition 3.8. This completes the proof of (1.15).

We next recall that $I_{d}(v)$ of (1.12) is strictly increasing in $v \geq 2 /(d-2)$, whereas $I_{d}\left(C_{d}\right)=2$. Hence, fixing $a>C_{d}$, we may and shall fix $\gamma>0$ such that $I_{d}(a)-2-\gamma>0$. Then, by (9.2), for any $\delta>0$ sufficiently small,

$$
\sum_{n=1}^{\infty} \mathbf{P}\left(\left|\mathscr{H}_{n}\right| \geq 1\right) \leq \sum_{n=1}^{\infty} \mathbb{E}\left|\mathscr{H}_{n}\right| \leq c_{1} \sum_{n=1}^{\infty} \eta_{n}^{I_{d}(a)-2-\gamma}<\infty .
$$

Thus, by Borel-Cantelli, it follows that a.s. $\mathscr{H}_{n}$ is empty for all $n \geq m_{2}(\omega)$, implying that the sets $D_{a}$ are a.s. empty for all $T<\infty$. Since a.s.

$$
\liminf _{\varepsilon \rightarrow 0} \frac{\mu_{\infty}^{W}(B(0, \varepsilon))}{\varepsilon^{2}}=0
$$

(see [29], Theorem 6.8), taking $k \uparrow \infty$ and $a \downarrow C_{d}$ completes the proof of (1.16), and hence of Theorem 1.6, subject only to (9.2).

The first step in the proof of (9.2) is the following simple lemma (see [21] for the definition and properties of Bessel processes).

LEMMA 9.1. Let $Z=\int_{0}^{T} U_{s}^{-2} d s$ with $\left\{U_{s}: s \in[0, T]\right\}$ the Bessel process of index $d^{\prime}=d / 2-1>0$, starting at $U_{0}=u \in(0, k]$. Then, for any $\alpha \in\left(0, d^{\prime}\right]$, $b>1$, there exist $c=c\left(b, T, d^{\prime}, k\right)<\infty$ such that

$$
\mathbb{E}_{\left(d^{\prime}\right)}^{u}\left(\exp \left(\left(d^{\prime 2} / 2-\alpha^{2} / 2\right) Z\right) \mathbf{1}_{\inf _{s \in[0, T]} U_{s} \leq v}\right) \leq c v^{2 \alpha / b} u^{-\left(d^{\prime}-\alpha\right)-2 \alpha / b} .
$$


Proof. Let $P_{(\nu)}^{u}(\cdot)$ denote the law of the Bessel process $\left\{U_{s}: s \in[0, T]\right\}$ of index $\nu>0$ starting at $U_{0}=u$. Recall that for any index $\nu>0$,

$$
d U_{s}=(\nu+1 / 2) \frac{d s}{U_{s}}+d B_{s}, \quad U_{0}=u>0,
$$

where $B_{s}$ is a one-dimensional Brownian motion. In particular, $d P_{\left(d^{\prime}\right)}^{u} / d P_{(\alpha)}^{u}$ exists for any $d^{\prime} \geq \alpha>0$ and is given by the Girsanov transformation as (see [21], page 419)

$$
\frac{d P_{\left(d^{\prime}\right)}^{u}}{d P_{(\alpha)}^{u}}=\left(\frac{U_{T}}{u}\right)^{d^{\prime}-\alpha} \exp \left(-\left(d^{\prime 2} / 2-\alpha^{2} / 2\right) \int_{0}^{T} U_{s}^{-2} d s\right) .
$$

In particular, by Hölder's inequality, for $q=b /(b-1)$,

$$
\begin{aligned}
& \mathbb{E}_{\left(d^{\prime}\right)}^{u}\left(\exp \left(\left(d^{\prime 2} / 2-\alpha^{2} / 2\right) Z\right) \mathbf{1}_{\inf _{s \in[0, T]} U_{s} \leq v}\right) \\
& \quad=u^{\alpha-d^{\prime}} \mathbb{E}_{(\alpha)}^{u}\left(U_{T}^{d^{\prime}-\alpha} \mathbf{1}_{\inf _{s \in[0, T]} U_{s} \leq v}\right) \\
& \leq u^{\alpha-d^{\prime}} P_{(\alpha)}^{u}\left(\inf _{s \geq 0} U_{s} \leq v\right)^{1 / b} \mathbb{E}_{(\alpha)}^{u}\left(U_{T}^{q\left(d^{\prime}-\alpha\right)}\right)^{1 / q} \\
& \leq u^{\alpha-d^{\prime}}\left(\frac{v}{u}\right)^{2 \alpha / b} \mathbb{E}_{(\alpha)}^{k}\left(U_{T}^{q\left(d^{\prime}-\alpha\right)}\right)^{1 / q}
\end{aligned}
$$

where the last inequality follows using the fact that the Bessel process of in$\operatorname{dex} \alpha$ has scale function $-x^{-2 \alpha}$ ([21], page 415), and for the rightmost expectation we used a simple comparison argument. A further comparison argument shows that we can take $c=\mathbb{E}_{\left(d^{\prime}\right)}^{k}\left(\left(1 \vee U_{T}\right)^{q d^{\prime}}\right)^{1 / q}<\infty$.

The next step in proving (9.2) is to establish the following consequence of Lemma 9.1.

LeMma 9.2. For any $T<\infty, b>1, k>1$, there exists $c<\infty$ such that for any $a>0, \alpha \in\left(0, d^{\prime}\right], \eta>0, \delta=\eta^{1-b^{-1}},|x| \in(0, k]$,

$$
\mathbf{P}\left(\inf _{\varepsilon \in[\eta, \delta]} \frac{\mu_{T}^{W}(B(x, b \varepsilon))}{\varepsilon^{2}} \geq \frac{a}{b}\right) \leq c \eta^{a b^{-4}\left(d^{2}-\alpha^{2}\right)+2 \alpha / b}|x|^{-\left(d^{\prime}-\alpha\right)-2 \alpha / b} .
$$

Proof. Fix $T, a, b, k, \alpha, \eta, \delta$ and $x$ as in the statement of the lemma. Observe that $U_{s}=\left|W_{s}-x\right|$ is a Bessel process of index $d^{\prime}$, starting at $U_{0}=|x| \in(0, k]$. Clearly,

$$
\left\{\mu_{T}^{W}(B(x, v))>0\right\}=\left\{\inf _{s \in[0, T]} U_{s}<v\right\} .
$$

Setting $Z=\int_{0}^{T} U_{s}^{-2} d s$, also

$$
\begin{aligned}
b^{2} Z & =\int_{0}^{T} \int_{b^{-1} U_{s}}^{\infty} \frac{2 d \varepsilon}{\varepsilon^{3}} d s=\int_{0}^{T} \int_{0}^{\infty} \mathbf{1}_{\left\{\left|W_{s}-x\right| \leq b \varepsilon\right\}} \frac{2 d \varepsilon}{\varepsilon^{3}} d s \\
& =\int_{0}^{\infty} \frac{2 d \varepsilon}{\varepsilon^{3}} \mu_{T}^{W}(B(x, b \varepsilon)) \geq \int_{\eta}^{\delta} \frac{2 d \varepsilon}{\varepsilon^{3}} \mu_{T}^{W}(B(x, b \varepsilon)) .
\end{aligned}
$$


If

$$
\inf _{\varepsilon \in[\eta, \delta]} \varepsilon^{-2} \mu_{T}^{W}(B(x, b \varepsilon)) \geq \frac{a}{b}
$$

then $\mu_{T}^{W}(B(x, b \eta))>0$ and

$$
\int_{\eta}^{\delta} \frac{2 d \varepsilon}{\varepsilon^{3}} \mu_{T}^{W}(B(x, b \varepsilon)) \geq \frac{a}{b} \int_{\eta}^{\delta} \frac{2 d \varepsilon}{\varepsilon}=-2 a b^{-2} \log \eta .
$$

Thus, for $v=b \eta$ and $\lambda=\left(d^{\prime 2}-\alpha^{2}\right) / 2 \geq 0$, by (9.9), (9.10) and Chebyshev's inequality,

$$
\begin{aligned}
\mathbf{P}\left(\inf _{\varepsilon \in[\eta, \delta]} \frac{\mu_{T}^{W}(B(x, b \varepsilon))}{\varepsilon^{2}} \geq \frac{a}{b}\right) & \leq P_{\left(d^{\prime}\right)}^{|x|}\left(Z \geq-2 a b^{-4} \log \eta, \inf _{s \in[0, T]} U_{s} \leq v\right) \\
& \leq \eta^{2 \lambda a b^{-4}} \mathbb{E}_{\left(d^{\prime}\right)}^{|x|}\left[\exp ^{\lambda Z} \mathbf{1}_{\inf _{s \in[0, T]} U_{s} \leq v}\right] .
\end{aligned}
$$

We thus obtain (9.8) by applying Lemma 9.1 .

We now return to complete the proof of (9.2). For $b>1$ and $\alpha \in\left(0, d^{\prime}\right]$ let

$$
f_{a}(b, \alpha)=a b^{-4}\left(d^{\prime 2}-\alpha^{2}\right)-d+2 \alpha / b .
$$

By Lemma 9.2, for some $c, c_{1}, c_{2}<\infty$ independent of $n$,

$$
\begin{aligned}
\mathbb{E}\left|\mathscr{H}_{n}\right| & =\sum_{j=1}^{K_{n}} \mathbf{P}\left(\inf _{\varepsilon \in\left[\eta_{n}, \delta_{n}\right]} \frac{\mu_{T}^{W}\left(B\left(x_{j}, b \varepsilon\right)\right)}{\varepsilon^{2}} \geq \frac{a}{b}\right) \\
& \leq c \eta_{n}^{a b^{-4}\left(d^{\prime 2}-\alpha^{2}\right)+2 \alpha / b} \sum_{j=1}^{K_{n}}\left|x_{j}\right|^{-\left(d^{\prime}-\alpha\right)-2 \alpha / b} \\
& \leq c_{1} \eta_{n}^{a b^{-4}\left(d^{\prime 2}-\alpha^{2}\right)+2 \alpha / b-d}\left(1+\int_{\{|x| \leq k\}}|x|^{-\left(d^{\prime}-\alpha\right)-2 \alpha / b} d x\right) \\
& \leq c_{2} \eta_{n}^{f_{a}(b, \alpha)},
\end{aligned}
$$

using $\left(d^{\prime}-\alpha\right)+2 \alpha / b<d^{\prime}+\alpha \leq d-1$.

Setting $\alpha=d^{\prime}-\theta$ for $\theta \in\left[0, d^{\prime}\right)$, in which case $d^{2}-\alpha^{2}=\theta(d-2-\theta)$, we see that

$$
f_{a}(b, \alpha)=a b^{-4} \theta(d-2-\theta)-d\left(1-b^{-1}\right)-(2 \theta+2) / b .
$$

Observe that $I_{d}(a)$, defined in (1.12), can also be written as

$$
(\max \{0, a(d-2)-2\})^{2} / 4 a,
$$

whence

$$
I_{d}(a)=\sup _{0 \leq \theta<(d-2) / 2}\{a \theta(d-2-\theta)-2 \theta\}
$$


and the supremum in (9.13) is attained at $\theta=\max \left\{0,(d-2) / 2-a^{-1}\right\}$. Comparing this with (9.12) we see that

$$
\lim _{b \downarrow 1} \sup _{\alpha \in\left(0, d^{\prime}\right]} f_{a}(b, \alpha)=I_{d}(a)-2,
$$

which completes the proof of (9.2) and hence of Theorem 1.6.

\section{SOME UNSOLVED PROBLEMS.}

1. Determine exactly the dimension appearing in (1.14) and the precise asymptotics in (1.16).

2. Does the set considered in (1.14) have equal Hausdorff and packing dimensions?

3. By arguments similar to those in the proof of (1.16), we can show that there exist nonrandom constants $\tilde{c}_{d}>0, \tilde{C}_{d}<\infty$ such that

$$
\tilde{c}_{d} \leq \inf _{t \in[0,1]} \limsup _{\varepsilon \rightarrow 0} \frac{\mu_{\infty}^{W}\left(B\left(W_{t}, \varepsilon\right)\right)}{\varepsilon^{2}} \leq \tilde{C}_{d} \quad \text { a.s. }
$$

More precisely, the upper bound here is proved just like the lower bound in (1.16), while the lower bound can be inferred from [17] or from a branching process argument. As in (1.16), it is an open problem to determine the optimal constants in (9.15).

Acknowledgments. We thank N.-R. Shieh and S. J. Taylor for useful discussions and for providing us with a preprint of [24] and J. Pitman for a useful discussion of the Ciesielski-Taylor identity. We are indebted to MSRI, to the organizers of the program on stochastic analysis there and in particular to S. Evans and R. Williams, for making our collaboration possible.

\section{REFERENCES}

[1] Barlow, M. T. and Perkins, E. (1984). Levels at which every Brownian excursion is exceptional. Seminar on Probability XVIII. Lecture Notes in Math. 1059 1-28. Springer, Berlin.

[2] Benjamini, I. and Peres, Y. (1994). Tree-indexed random walks on groups and first passage percolation. Probab. Theory Related Fields 98 91-112.

[3] Ciesielski, Z. and Taylor, S. J. (1962). First passage and sojourn times and the exact Hausdorff measure of the sample path. Trans. Amer. Math. Soc. 103 434-452.

[4] Deheuvels, P. and Mason, D. M. (1998). Random fractal functional laws of the iterated logarithm. Studia Sci. Math Hungar. 34 89-106.

[5] Dembo, A., Peres, Y., Rosen, J. and Zeitouni, O. (1999). Thick points for transient symmetric stable processes. Elect. J. Probab. 4 1-18.

[6] Dembo, A., Peres, Y., Rosen, J. and Zeitouni, O. (1999) Thick points for planar Brownian motion and the Erdos-Taylor conjecture on random walk. Acta Math. To appear.

[7] Dembo, A., Peres, Y., Rosen, J. and Zeitouni, O. (1999). Thin points for Brownian motion. Ann. Inst. H. Poincaré Math. Statist. Probab. To appear.

[8] Falconer, K. J. (1990). Fractal Geometry: Mathematical Foundations and Applications. Wiley, New York.

[9] Hu, X. and Taylor, S. J. (1997). The multifractal structure of stable occupation measure. Stochastic Process. Appl. 66 283-299. 
[10] Kaufman, R. (1969). Une propriété metriqué du mouvement brownien. C. R. Acad. Sci. Paris 268 727-728.

[11] Lawler, G. The frontier of a Brownian path is multifractal. Preprint.

[12] Olsen, L. (1995). A multifractal formalism. Adv. Math. 116 82-196.

[13] Orey, S. and Taylor, S. J. (1974). How often on a Brownian path does the law of the iterated logarithm fail? Proc. London Math. Soc. 28 174-192.

[14] Pemantle, R. and Peres, Y. (1995). Galton-Watson trees with the same mean have the same polar sets. Ann. Probab. 23 1102-1124.

[15] Pemantle, R., Peres, Y. and Shapiro, J. W. (1996). The trace of spatial Brownian motion is capacity-equivalent to the unit square. Probab. Theory Related Fields 106 379-399.

[16] Peres, Y. (1996). Intersection-equivalence of Brownian paths and certain branching processes. Comm. Math. Phys. 177 417-443.

[17] Perkins, E. A. (1983). On the Hausdorff dimension of Brownian slow points. Z. Wahrsch. Verw. Gebeite 64 369-399.

[18] Perkins, E. A. and Taylor, S. J. (1987). Uniform measure results for the image of subsets under Brownian motion. Probab. Theory Related Fields 76 257-289.

[19] Perkins, E. A. and Taylor, S. J. (1988). Measuring close approaches on a Brownian path. Ann. Probab. 16 1458-1480.

[20] Perkins, E. A. and Taylor, S. J. (1998). The multifractal structure of super-Brownian motion. Ann. Inst. H. Poincaré Math. Statist. Probab. 34 97-138.

[21] Revuz D. and Yor, M. (1991) Continuous Martingales and Brownian Motion. Springer, New York.

[22] Reidi, R. (1995). An improved multifractal formalism and self-similar measures. J. Math. Anal. Appl. 189 462-490.

[23] Rogers, C. A. and Taylor, S. J. (1961). Functions continuous and singular with respect to a Hausdorff measure. Mathematika 8 1-31.

[24] Shieh, N.-R. and Taylor, S. J. (1998). Logarithmic multifractal spectrum of stable occupation measure. Stochastic Process. Appl. 75 249-269. [Correction (1999). Trends in Probability and Related Analysis 147-158. World Scientific, Singapore.

[25] Stroock, D. W. (1993). Probability Theory, an Analytic View. Cambridge Univ. Press.

[26] Taylor, S. J. (1974). Regularity of irregularities on a Brownian path. Ann. Inst. Fourier (Grenoble) 39 195-203.

[27] Taylor, S. J. (1986). The measure theory of random fractals. Math. Proc. Cambridge Philos. Soc. 100 383-406.

[28] Taylor, S. J. (1995). Super Brownian motion is a fractal measure for which the multifractal formalism is invalid. In Symposium in Honor of Benoit Mandelbrot 3 737-746 (Curagao).

[29] Taylor, S. J. and Tricot, C. (1985). Packing measure and its evaluation for a Brownian path. Trans. Amer. Math. Soc. 288 679-699.

[30] Tricot, C. (1982). Two definitions of fractal dimension. Math. Proc. Cambridge Philos. Soc. 91 57-74.

[31] Watson, G. N. (1922). A Treatise on the Theory of Bessel Functions. Cambridge Univ. Press.

\author{
A. Dembo \\ DePARTMENTS OF MATHEMATICS \\ AND OF STATISTICS \\ STANFORD UNIVERSITY \\ STANFORD, CALIFORNIA 94305 \\ E-MAIL: amir@math.stanford.edu

\section{J. ROSEN} \\ Department of Mathematics \\ College of Staten Island, CUNY \\ STATEN IsLAND, NEW YoRK 10314 \\ E-MAIL: jrosen3@idt.net
}

\author{
Y. PERES \\ DEPARTMENT OF STATISTICS \\ UNIVERSITY OF CALIFORNIA \\ BERKELEY, CALIFORNIA 94720 \\ E-MAIL: peres@stat.berkeley.edu
}

\section{O. Zeitouni}

Department of Electrical ENGINEERING TECHNION

HAIFA 32000, ISRAEL

E-MAIL: zeitouni@ee.technion.ac.il 